\title{
Functions of several Cayley-Dickson variables and manifolds over them.
}

\author{
S.V. Lüdkovsky
}

31 May 2004

\begin{abstract}
Functions of several octonion variables are investigated and integral representation theorems for them are proved. With the help of them solutions of the $\tilde{\partial}$-equations are studied. More generally functions of several Cayley-Dickson variables are considered. Integral formulas of the Martinelli-Bochner, Leray, Koppelman type used in complex analysis here are proved in the new generalized form for functions of Cayley-Dickson variables instead of complex. Moreover, analogs of Stein manifolds over Cayley-Dickson graded algebras are defined and investigated.
\end{abstract}

\section{Introduction}

In previous papers functions of one quaternion and Cayley-Dickson variables were investigated $[19,20]$. In them superdifferentiability of functions was studied and the theory of holomorphic functions was investigated. It was done with the help of line integration introduced and studied there. This line integration restricted on complex functions gives ordinary Cauchy integral, but ordinary Cauchy integral can not be in the usual manner extended on continuous functions of Cayley-Dickson numbers.

This line integral is additive by rectifiable paths and continuous functions in open domains in Cayley-Dickson algebras $\mathcal{A}_{p}$, it is also $\mathbf{R}$-homogeneous and left and right linear over quaternions, but generally nonlinear relative to quaternions or octonions or Cayley-Dickson algebras of higher order. Over Cayley-Dickson algebras of higher order than quaternions left or right linearity of operators certainly undermines relations between ordered (associated) products of generators of Cayley-Dickson numbers, for example, $(i j) l=-i(j l)$ for generators of the octonion algebra $\mathbf{O}$, where $\{1, i, j, k\}$ are standard generators of the quaternion algebra $\mathbf{H}, l$ is the generator of doubling procedure of the construction of $\mathbf{O}$ from $\mathbf{H}$ $[1,12]$. The line integral over Cayley-Dickson algebras for $z$-superdifferentiable (that is, $\mathcal{A}_{p}$-holomorphic) functions in corresponding domains $U$ depends only on specific homotopy classes of rectifiable paths with given initial and final points and also satisfies at least locally $\left(\partial\left[\int_{\gamma_{z}} f(\xi) d \xi\right] / \partial z\right) .1=f(z)$, for example, in a ball $B$ in $U$, where $\gamma_{z}:[0,1] \rightarrow U, \gamma(0)$ and $\gamma_{z}(1)=z \in B$.

The Cayley-Dickson algebra is $\mathbf{Z}_{\mathbf{2}}$-graded, that is, superalgebra. In the theory of superalgebras it was traditionally used the notion of superdifferentiabilty (left or right superlinear) [8]. It causes strong restrictions on the types of admissible functions. For example, over Grassman algebras it produces functions only linear in odd arguments $[2,4]$. In general it leads to conditions analogous to Cauchy-Riemann. 
Cayley-Dickson algebras such as quaternion and octonion algebras have found applications in quantum mechanics and noncommutative geometry [3, 5, 13, 21, 24]. The latter is especially valuable in conjunction with operator algebras, which permits to consider quantization. On the other hand, Cayley-Dickson algebras are not central over the field of complex numbers C. Moreover, the octonion algebra and Cayley-Dickson algebras of higner order can not be written as matrices with entries in the field of real or complex numbers, though their centre is $\mathbf{R}$.

It is necessary to note, that apart from the real or complex case derivatives of superdifferentiable functions of Cayley-Dickson numbers are operators even in the case of one variable. Therefore, a superderivative of the line integral by a final point of the path is an operator. To work with rings of superdifferentiable functions it was introduced the condition of $\mathcal{A}_{p^{-}}$ additivity instead of right or left superlinearity of a superdifferential $[19,20]$. It is natural, since if to start from the family $\mathcal{F}$ of all right superlinearly superdifferentiable functions $f: U \rightarrow \mathcal{A}_{p}, p \geq 2$, then the using of Leibnitz rule for finite ordered (associated) products $\left\{f_{1}, \ldots, f_{m}\right\}_{q(m)}$ of $f_{1}, \ldots, f_{m} \in \mathcal{F}$ gives only $\mathbf{R}$-homogeneous $\mathcal{A}_{p}$-additive superdifferential, where $U$ is open in $\mathcal{A}_{p}^{n}$, a vector $q(m)$ indicates on the order of multiplication. This superdifferential operator can be extended on the corresponding family of converging series arising from such final products and hence on locally analytic functions of $z_{1}, \ldots, z_{n} \in \mathcal{A}_{p}$. Since there are many embeddings of $\mathbf{C}$ into $\mathcal{A}_{p}, p \geq 2$, then to encompass the case of complex holomorphic functions in such theory it was introduced the condition analogous to holomorphicity: $\partial f / \partial \tilde{z}=0$, where $z \tilde{z}=|z|^{2}$, $\tilde{z}$ denotes the adjoint of a Cayley-Dickson number $z$.

To make it accurately it was used the notions of words and phrases and germs of functions and the Stone-Weierstrass theorem. On the other hand, such formalized definition of superdifferentiability does not impose from the beginning the condition of local analyticity. It was proved in $[19,20]$ under definite conditions equivalence of Cayley-Dickson holomorphicity, independence of the line integral over $\mathcal{A}_{p}$ of holomorphic function from the rectifiable paths with common beginning and final points, local analyticity of functions. If $f: U \rightarrow \mathcal{A}_{p}$ is superdifferentiable, where $U$ is open in $\mathcal{A}_{p}^{n}$, then there exists an $\mathcal{A}_{p}$-superdifferentiable $g: U \rightarrow \mathcal{A}_{p}$ such that $(\partial g(z) / \partial z) .1=f(z)$ and $\hat{f}(z):=(\partial g(z) / \partial z)$. Then the path integral is defined with the help of the operator $\hat{f}$. The line integral along each rectifiable path $\gamma$ has the continuous extension from $C^{1}\left(U, \mathcal{A}_{p}\right)$ onto $C^{0}\left(U, \mathcal{A}_{p}\right)$. Thus there can be given the following.

1. Definitions. Define $\hat{f}$ as a generalized function for each $f \in C^{0}\left(U, \mathcal{A}_{p}\right)$ in the sense of distributions: $(\phi, \hat{f})_{\gamma}:=\int_{\gamma} \phi(z) f(z) d z$, where $\phi$ is infinite $\mathcal{A}_{p}$-superdifferentiable, $\gamma$ is a rectifiable path in a subset $U$ open in $\mathcal{A}_{p}^{n}$. Denote $\hat{f}^{(0)}(z):=\hat{f}(z)$. Define the $k$-th derivative $\left(\phi, \hat{f}^{(k)} \cdot\left({ }^{1} h, \ldots,{ }^{k} h\right)\right)_{\gamma}:=(-1)^{k}\left(\phi^{(k)} \cdot\left({ }^{1} h, \ldots,{ }^{k} h\right), \hat{f}\right)_{\gamma}$ for each $\gamma$ and each infinite $\mathcal{A}_{p}$-superdifferentiable $\phi$ on $U$ and equal to zero in neighborhoods of $\gamma(0)$ and $\gamma(1)$, where $\gamma:[0,1] \rightarrow U$ is a rectifiable path, ${ }^{1} h, \ldots,{ }^{k} h \in \mathcal{A}_{p}, 0<k \in \mathbf{N}$. A net $\left\{\hat{f}_{\alpha}^{(k)}: \alpha \in \mathcal{Y}\right\}$ converges to $\hat{f}^{(k)}$ on $U$ in the sense of distributions, if the net $\left(\phi^{(k)} .\left({ }^{1} h, \ldots,{ }^{k} h\right), \hat{f}_{\alpha}\right)_{\gamma}$ converges for each infinite $\mathcal{A}_{p}$-superdifferentiable function $\phi$ on $U$ and each rectifiable path $\gamma$ in $U$ and each ${ }^{1} h, \ldots,{ }^{k} h$, where $0 \leq k \in \mathbf{Z}, \phi$ has the corresponding support, $\mathcal{Y}$ is a directed set. The same type of generalized derivatives of a continuous function $f$ and their convergence in the sense of distributions we adopt for $f$ instead of $\hat{f}$ using the identity $f^{(k)}(z)=\hat{f}^{(k)}(z) .1$.

This article is devoted to functions of several Cayley-Dickson variables and investigations of integral representation formulas for them. Moreover, such formulas are also obtained for differential forms over $\mathcal{A}_{p}, p \geq 2$, where $\mathcal{A}_{2}=\mathbf{H}, \mathcal{A}_{3}=\mathbf{O}$. There are well-known integral formulas of the Martinelli-Bochner, Leray, Koppelman type widely used in complex analysis. 
Here new generalized formulas are proved for functions of Cayley-Dickson variables instead of complex. Moreover, analogs of Stein manifolds over Cayley-Dickson graded algebras are defined and investigated.

The results of this paper it is possible to apply for further investigations of transformation (super)groups and corresponding to them (super)algebras of manifolds over Cayley-Dickson algebras as well as loop spaces, measures and stochastic processes on them, continuing previous studies of groups of loops and groups of diffeomorphisms of Riemannian and complex manifolds $[15,16,17,18]$, for they are widely used in mathematical physics and gauge theories.

\section{Differentiable functions of several Cayley-Dickson vari- ables}

2.1. Theorem. Let $U$ be an open subset in $\mathcal{A}_{r}, 2 \leq r<\infty$, with a $C^{1}$-boundary $\partial U$ $U$-homotopic with a product $\gamma_{1} \times \gamma_{2} \times \ldots \times \gamma_{m}$, where $m=2^{r}-1, \gamma_{j}(\theta)=a_{j}+\rho_{j} \exp \left(2 \pi \theta M_{j}\right)$, $M_{j} \in \mathcal{I}_{r},\left|M_{j}\right|=1, \theta \in[0,1], \gamma_{j}([0,1]) \subset U, 0<\rho_{j}<\infty, j=1,2, \ldots, m$, where $M_{1}, \ldots, M_{m}$ are linearly independent over $\mathbf{R}$. Let also $f: \operatorname{cl}(U) \rightarrow \mathcal{A}_{r}$ be a continuous function on cl $(U)$ such that $(\partial f(z) / \partial \tilde{z})$ is defined in the sense of distributions in $U$ is continuous in $U$ and has a continuous extension on $\mathrm{cl}(U)$, where $U$ and $\gamma_{j}$ for each $j$ satisfy conditions of Theorem 3.9 [20], where $\mathcal{I}_{r}:=\left\{z \in \mathcal{A}_{r}: z+\tilde{z}=0\right\}$. Then

$$
\begin{gathered}
\text { (1) } f(z)=(2 \pi)^{-m} \int_{\gamma_{m}}\left(\int _ { \gamma _ { m - 1 } } \left(\ldots \left(\int_{\gamma_{2}}\left(\int_{\gamma_{1}} f\left(\zeta_{1}\right) \cdot\left[\left(\partial_{\zeta_{1}} \operatorname{Ln}\left(\zeta_{1}-\zeta_{2}\right)\right) M_{1}^{*}\right]\right) \cdot[\right.\right.\right. \\
\left.\left.\left.\left(\partial_{\zeta_{2}} \operatorname{Ln}\left(\zeta_{2}-\zeta_{3}\right)\right) M_{2}^{*}\right]\right) \ldots\left[\left(\partial_{\zeta_{m-1}} \operatorname{Ln}\left(\zeta_{m-1}-\zeta_{m}\right)\right) M_{m-1}^{*}\right]\right) \ldots\left[\partial_{\zeta_{m}} \operatorname{Ln}\left(\zeta_{m}-z\right)\right) M_{m}^{*} \\
\quad-(2 \pi)^{-m} \int_{U}\left\{\left(\ldots\left(\left(\left(\left(\partial \hat{f}\left(\zeta_{1}\right) / \partial \tilde{\zeta}_{1}\right) \cdot d \tilde{\zeta}_{1}\right) \wedge \partial_{\zeta_{1}} \operatorname{Ln}\left(\zeta_{1}-\zeta_{2}\right)\right) M_{1}^{*}\right)\right.\right. \\
\left.\left.\left.\wedge\left(\partial_{\zeta_{2}} \operatorname{Ln}\left(\zeta_{2}-\zeta_{3}\right)\right) M_{2}^{*}\right) \wedge \ldots\right) \wedge\left(\partial_{\zeta_{m}} \operatorname{Ln}\left(\zeta_{m}-z\right)\right) M_{m}^{*}\right\}, \quad m:=2^{p}-1 .
\end{gathered}
$$

Proof. We have the identities $d_{\zeta}\left[\hat{f}(\zeta) \cdot \partial_{\zeta} \operatorname{Ln}(\zeta-z)\right]=\{(\partial \hat{f}(\zeta) / \partial \tilde{\zeta}) \cdot d \tilde{\zeta}\} \wedge \partial_{\zeta} \operatorname{Ln}(\zeta-z)$ $+\{(\partial \hat{f}(\zeta) / \partial \zeta) \cdot d \zeta\} \wedge \partial_{\zeta} \operatorname{Ln}(\zeta-z)$ and $\left.d_{\zeta} d_{\zeta} \operatorname{Ln}(\zeta-z)\right|_{\zeta \in \gamma}=0$ for $\zeta$ varying along a path $\gamma$, where for short $f(z)=f(z, \tilde{z})$, since there is the bijection of $z$ with $\tilde{z}$ on $\mathcal{A}_{r}$. There exists $\mathbf{R}$ homogeneous $\mathcal{A}_{r}$-additive operator-valued function $q(\zeta, z)$ such that $\partial_{\zeta} \operatorname{Ln}(\zeta-z)=q(\zeta, z) . d \zeta$ (see also $\S \S 2.1,2.2,2.6$ and $2.7[20])$. As in $[19,20] \hat{f}(z, \tilde{z}):=\partial g(z, \tilde{z}) / \partial z$, where $g(z, \tilde{z})$ is an $\mathcal{A}_{r}$-valued function such that $(\partial g(z, \tilde{z}) / \partial z) .1=f(z, \tilde{z})$. Since $\zeta_{1}$ varies along the path $\gamma_{1}$, then $\left.d \zeta_{1} \wedge d \zeta_{1}\right|_{\zeta_{1} \in \gamma_{1}}=0$. Consider $z \in U$ and $\epsilon>0$ such that the torus $\mathbf{T}\left(z, \epsilon, \mathcal{A}_{r}\right)$ is contained in $U$, where $\partial \mathbf{T}\left(z, \epsilon, \mathcal{A}_{r}\right)=\psi_{m} \times \ldots \times \psi_{2} \times \psi_{1}, \psi_{j}$ are of the same form as $\gamma_{j}$ but with $z$ instead of $a_{j}$ and with $\rho_{j}=\epsilon$. Applying Stokes formula for regions in $\mathbf{R}^{2^{r}}$ and componentwise to $\mathcal{A}_{r}$-valued differential forms we get

$$
\begin{gathered}
\int_{\partial U} \omega-\int_{\partial \mathbf{T}\left(z, \epsilon, \mathcal{A}_{r}\right)} \omega=\int_{U \backslash \mathbf{T}\left(z, \epsilon, \mathcal{A}_{r}\right)} d w, \text { where } \\
\left.w=\left(\ldots\left(\left(\hat{f}\left(\zeta_{1}\right) \cdot\left[\left(\partial_{\zeta_{1}} \operatorname{Ln}\left(\zeta_{1}-\zeta_{2}\right)\right) M_{1}^{*}\right]\right) \cdot\left[\left(\partial_{\zeta_{2}} \operatorname{Ln}\left(\zeta_{2}-\zeta_{3}\right)\right) M_{2}^{*}\right]\right) \ldots\right) \cdot\left[\left(\partial_{\zeta_{m}} \operatorname{Ln}\left(\zeta_{m}-z\right)\right) M_{m}^{*}\right]\right),
\end{gathered}
$$

$m=2^{r}-1$. Then $(d L n \exp (\theta M)) M^{*}=d \theta$ and $\hat{f}(z) \cdot d \theta=f(z) d \theta$, since $M \in \mathcal{I}_{r}, M \neq 0$ and $\theta \in \mathbf{R}$. In view of Theorems 3.9 and 3.23 [20] we have that

$\lim _{\epsilon \rightarrow 0, \epsilon>0}(2 \pi)^{-m} \int_{\gamma_{m}}\left(\ldots\left(\int_{\gamma_{2}}\left(\int_{\gamma_{1}} w\right)\right) \ldots\right)=f(z)$ and 
$\lim _{\epsilon \rightarrow 0, \epsilon>0} \int_{U \backslash \mathbf{T}\left(z, \epsilon, \mathcal{A}_{r}\right)} d \omega=\int_{U} d \omega$.

From this formula (1) follows.

2.1.1. Remark. Formula (2.1) is the Cayley-Dickson algebras' analog of the (complex) Cauchy-Green formula. Since in the sence of distributions $\partial \hat{f} / \partial \tilde{z}=\partial(\partial g / \partial \tilde{z}) / \partial z$ (see Definition 1), then from $\partial \hat{f} / \partial \tilde{z}=0$ it follows, that $\partial \hat{f} .1 / \partial \tilde{z}=\partial f / \partial \tilde{z}=0$. If $\partial f / \partial \tilde{z}=0$, then $g$ can be chosen such that $\partial g / \partial \tilde{z}=0$ [20]. Therefore, from Formula (2.1) it follows, that $f$ is $\mathcal{A}_{r}$-holomorphic in $U$ if and only if $\partial f / \partial \tilde{z}=0$ in $U$.

2.2.1. Remark. Instead of curves $\gamma$ of Theorem 2.1 above or Theorems 3.9, 3.23 [20] it is possible to consider their natural generalization $\gamma(\theta)+z_{0}=z_{0}+\rho(\theta) \exp (2 \pi S(\theta))$, where $\rho(\theta)$ and $S(\theta)$ are continuous functions of finite total variations, $\theta \in[0,1] \subset \mathbf{R}, \rho(\theta) \geq 0$, $S(\theta) \in \mathcal{I}_{r}, 2 \leq r \leq \infty$. Therefore, $\gamma$ is a rectifiable path. If $S(0)=S(1) \bmod \left(\mathcal{S}_{r}\right)$ and $\rho(0)=\rho(1)$, then $\gamma$ is a closed path (loop): $\gamma(0)=\gamma(1)$, where $\mathcal{S}_{r}:=\left\{z \in \mathcal{I}_{r}:|z|=1\right\}$, $\mathcal{I}_{r}:=\left\{z \in \mathcal{A}_{r}: z+\tilde{z}=0\right\}$. Consider $S$ absolutely continuous such that there exists $T \in L^{1}\left([0,1], \mathcal{A}_{r}\right.$ ) for which $S(\theta)=S(0)+\int_{0}^{\theta} T(\tau) d \tau$ (see Satz 2 and 3 (Lebesgue) in $\S 6.4[11]$ ) and let $\rho(\theta)>0$ for each $\theta \in[0,1]$. Evidently, $M n:=S(1)-S(0)=\int_{0}^{1} T(\tau) d \tau$ is invariant relative to reparatmetrizations $\phi \in \operatorname{Dif} f_{+}^{1}([0,1])$ of diffeomorphisms of $[0,1]$ preserving the orientation, $n$ is a real number, $M \in \mathcal{S}_{r}$. Then $\Delta \operatorname{Arg}(\gamma):=\left.\operatorname{Arg}(\gamma)\right|_{0} ^{1}=2 \pi \int_{0}^{1} T(\tau) d \tau$ (see also Formula (3.7) and $\$ 3.8 .3[20]$ ). In view of Theorem 3.8.3 [20] for each loop $\gamma$ : $\Delta \operatorname{Arg}(\gamma) \in \mathbf{Z} \mathcal{S}_{r}$. For each $\epsilon>0$ for the total variation there is the equality $V(\gamma \epsilon)=V(\gamma) \epsilon$. Since $\gamma([0,1])$ is a compact subset in $\mathcal{A}_{r}$, then there exists $\rho_{m}:=\sup _{\theta \in[0,1]} \rho(\theta)<\infty$. Hence $z_{0}+(\gamma \epsilon)([0,1]) \subset B\left(\mathcal{A}_{r}, z_{0}, \rho_{m} \epsilon\right)$.

Therefore, Theorems 3.9, 3.23, 3.28 and Formulas (3.9, 3.34.i) [20] and Theorem 2.1 above are true for such paths $\gamma$ also and Formula (3.9) [20] takes the form

$$
\text { (1) } f(z) M=(2 \pi n)^{-1}\left(\int_{\psi} f(\zeta)(\zeta-z)^{-1} d \zeta\right),
$$

where $0 \neq n \in \mathbf{Z}$ for a closed path $\gamma, M \in \mathcal{S}_{r}$, Formula (1) generalizes Formula (3.9), when $|n|>1$. When $\hat{I} n(0, \gamma)=0$, then $\left(\int_{\psi} f(\zeta)(\zeta-z)^{-1} d \zeta\right)=0$ (see also $\S 3.23[20]$ ).

2.2.2. Note and Definition. Let $\Lambda$ denotes a Hausdorff topological space with nonnegative measure $\mu$ on a $\sigma$-algebra of all Borel subsets such that for each point $x \in \Lambda$ there exists an open neighborhood $U \ni x$ with $0<\mu(U)<\infty$. Consider a set of generators with real algebra $\left\{i_{x}: x \in \Lambda\right\}$ such that $i_{x} i_{y}=-i_{y} i_{x}$ for each $x \neq y \in \Lambda \backslash\{0\}$ and $i_{x}^{2}=-1$ for each $x \in \Lambda \backslash\{0\}$, where 0 is a marked point in $\Lambda$. Add to this set the unit $1=: i_{0}$ such that $a i_{x}=i_{x} a$ for each $a \in \mathbf{R}$ and $x \in \Lambda$. In the case of a finite set $\Lambda$ the Cayley-Dickson algebra generated by such generators is isomorphic with $\mathcal{A}_{N-1}$, where $N=\operatorname{card}(\Lambda)$ is the cardinality of the set $\Lambda$.

For the infinite subset of generators $\left\{i_{0}, i_{x_{j}}: j \in \mathbf{N}, x_{j} \in \Lambda\right\}$ the construction from $\S 3.6 .1$ [20] produces the algebra isomorphic with $\mathcal{A}_{\infty}$. Therefore, consider the case $\operatorname{card}(\Lambda)>\aleph_{0}$. Due to the Kuratowski-Zorn lemma we can suppose, that $\Lambda$ is linearly ordered and this linear ordering gives intervals $(a, b):=\{x \in \Lambda: a<x<b\}$ being $\mu$-measurable, for example, $\Lambda=\mathbf{R}^{n} \times(\mathbf{R} / \mathbf{Z})^{m}$ has the natural linear ordering induced by the linear ordering from $\mathbf{R}$ and by the lexicographic ordering in the product, where $n, m \in \mathbf{N}$.

Then consider a finite partition $\Lambda$ into a disjoint union $\Lambda=\bigcup_{j=0}^{p} A_{j}$, where $x<y$ for each $x \in A_{j}$ and $y \in A_{l}$ for $j<l \leq p, p \in \mathbf{N}, 0 \in A_{0}$. The family of such partitions we denote $\mathcal{Z}$. Let $T \in \mathcal{Z}, x_{j} \in A_{j}$ be marked points. Then there exists a step function $f_{T}$ such that $f_{T}(x)=C_{j} i_{x_{j}}$ for each $x \in A_{j}$, where $C_{j} \in \mathbf{R}$. Consider the norm $\left\|f_{T}\right\|_{\Lambda}^{2}:=$ $\int_{\Lambda} f_{T}(x) \tilde{f}_{T}(x) \mu(d x)$, where $\tilde{f}_{T}(x):=C_{0} \chi_{A_{0}}(x) \delta_{0, x_{0}}-\sum_{x_{j} \neq 0} C_{j} \chi_{A_{j}}(x) i_{x_{j}}, \chi_{A}(x)=1$ for $x \in A$, $\chi_{A}(x)=0$ for $x \notin A, \delta_{x, y}=1$ for $x=y, \delta_{x, y}=0$ while $x \neq y$. To each $f_{T}$ put the element $z_{f_{T}}:=\sum_{j} C_{j} i_{x_{j}} \mu\left(A_{j}\right)$. 
The algebra which is the completion by the norm $\|*\|_{\Lambda}$ of the minimal algebra generated by the family of elements $z_{f_{T}}$ for $f_{T}$ from the family $\mathcal{F}$ of all step functions and all their ordered final products we denote by $\mathcal{A}_{\Lambda}$.

2.2.3. Theorem. The set $\mathcal{A}_{\Lambda}$ is the power-associative noncommutative nonassociative algebra over $\mathbf{R}$ complete relative to the norm $\|*\|_{\Lambda}$ with the centre $Z\left(\mathcal{A}_{\Lambda}\right)=\mathbf{R}$, moreover, there are embeddings $\mathcal{A}_{\infty} \hookrightarrow \mathcal{A}_{\Lambda}$ for $\operatorname{card}(\Lambda) \geq \aleph_{0}$. The set of generators of the algebra $\mathcal{A}_{\Lambda}$ has the cardinality card $(\Lambda)$ for $\operatorname{card}(\Lambda) \geq \operatorname{card}(\mathbf{N})$. There exists the function $\overrightarrow{\exp }\left(\int_{\Lambda} f(x) \mu(d x)\right)$ of the ordered integral product from $\mathcal{A}_{\Lambda}$ onto $\mathcal{A}_{\Lambda}$.

Proof. For $\operatorname{card}(\Lambda) \leq \aleph_{0}$ the algebra $\mathcal{A}_{\Lambda}$ is isomorphic with $\mathcal{A}_{N-1}$ or $\mathcal{A}_{\infty}$. Thus it remains to consider the case $\operatorname{card}(\Lambda)>\aleph_{0}$. For each $f_{T} \in \mathcal{F}$ it can be defined the ordered integral exponential product

$\overrightarrow{\exp }\left(\int_{\Lambda} f_{T}(x) \mu(d x)\right):=\left\{\exp \left(C_{0} \mu\left(A_{0}\right) \pi i_{x_{0}} / 2\right) \ldots \exp \left(C_{p} \mu\left(A_{p}\right) \pi i_{x_{p}} / 2\right)\right\}_{q(p+1)}$

with $q(p+1)$ corresponding to the left order of brackets. Thus there exist the embeddings of $\mathcal{A}_{\infty}$ into $\mathcal{A}_{\Lambda}$. Then $Z\left(\mathcal{A}_{\Lambda}\right)=\mathbf{R}$. The completion of the family $\mathcal{F}$ contains all functions of the type $f(x)=\sum_{j} f_{j}(x) \chi_{A_{j}}(x) i_{x_{j}}$, where $\left\{A_{j}: j \in \mathbf{N}\right\}$ is the disjoint union of $\Lambda$, each $A_{j}$ is $\mu$-measurable, $f_{j} \in L^{2}(\Lambda, \mu, \mathbf{R})$ and $\lim _{n \rightarrow \infty} \sum_{j>n}\left\|f_{j}(x) \chi_{A_{j}}(x)\right\|_{L^{2}(\Lambda, \mu, \mathbf{R})}^{2}=0$.

Since $\exp (M)=\cos (|M|)+M \sin (|M|) /|M|$ for each $M \in \mathcal{A}_{\infty}$ and $|\exp (M)-1| \leq$ $\exp (|M|)-1$, then for each $f \in \mathcal{A}_{\Lambda}$ there exists

$\lim _{\mathcal{F} \ni f_{T} \rightarrow f} \overrightarrow{\exp }\left(\int_{\Lambda} f_{T}(x) \mu(d x)\right)=: \overrightarrow{\exp }\left(\int_{\Lambda} f(x) \mu(d x)\right)$

relative to $\|*\|_{\Lambda}$. From $\exp \left(\pi i_{x} / 2\right)=i_{x}$ for each $x \in \Lambda \backslash\{0\}$ it follows, that the family of all elements of the type $\overrightarrow{\exp }\left(\int_{\Lambda} f(x) \mu(d x)\right), f \in \mathcal{F}$ contains all generators of the embedded subalgebra $\mathcal{A}_{\infty}$ generated by the countable subfamily $\left\{i_{x_{j}}: j \in \mathbf{N}\right\}$.

The completion $\tilde{\mathcal{F}}$ of the family $\mathcal{F}$ by the norm $\|*\|_{\Lambda}$ is the infinite dimensional linear subspace over $\mathbf{R}$ in $\mathcal{A}_{\Lambda}$. All possible final ordered products from $\tilde{\mathcal{F}}$ and the completion of their $\mathbf{R}$-linear span by the norm $\|*\|_{\Lambda}$ produces $\mathcal{A}_{\Lambda}$. Then for each element from $\mathcal{A}_{\Lambda}$ there exists the representation in the form of the ordered integral exponential product. Since $\mathcal{A}_{\Lambda}$ is the algebra over $\mathbf{R}$ and $\operatorname{card}(\Lambda)^{\aleph_{0}}=\operatorname{card}(\Lambda)$, then the family of generators of the algebra $\mathcal{A}_{\Lambda}$ has the cardinality $\operatorname{card}(\Lambda)$.

2.2.4. Note. Evidently Propositions 2.2.1,2.3, 2.6 and Corollary 2.4, Lemma 2.5.1 [20] are accomplished in the case of $\mathcal{A}_{\Lambda}$ with $\mathbf{b}=\mathbf{b}_{\Lambda}$ instead of $\mathbf{b}=\mathbf{b}_{r}$. Definition 2.5 has the meaning also for $\mathcal{A}_{\Lambda}$. Theorem 2.7 is also accomplished for $\mathcal{A}_{\Lambda}$, since for each $z \in \mathcal{A}_{\Lambda}$ there exists the embedded subalgebra isomorphic with $\mathcal{A}_{\infty}$ and containing $z$. A path $\gamma$ is rectifiable, hence it has a countable dense subset. For each $\epsilon>0$ there exists a subalgebra isomorphic with $\mathcal{A}_{\infty}$ the projection $\psi(t)$ on which of the path $\gamma$ differs from $\gamma(t)$ no more than on $\epsilon$ for each $t \in[a, b]$, where $\gamma:[a, b] \rightarrow \mathcal{A}_{\Lambda}$. For $\mathcal{A}_{\infty}$ with the help of the projections $P_{r}$ we have $\psi=\lim _{r \rightarrow \infty} P_{r}(\psi), P_{r}(\psi) \subset U_{r},\left\{P_{r}(\gamma): r \in \mathbf{N}\right\}$, converges to $\psi$ uniformly on the compact segment $[a, b] \subset \mathbf{R}$, where $U_{r}=P_{r}(U)$. Take a sequence of such path $\psi_{n}$ with $\sup _{t \in[a, b]}\left|\psi_{n}(t)-\gamma(t)\right|<1 / n$. Then $\int_{\psi_{n}} f(z) d z$ forms the Cauchy sequence in $\mathcal{A}_{\Lambda}$, which is complete. Thus there exists $\lim _{n \rightarrow \infty} \int_{\psi_{n}} f(z) d z=\int_{\gamma} f(z) d z$. Consequently, the integral along the path has the unique continuous extension on $C_{b}^{0}\left(U, \mathcal{A}_{\Lambda}\right)$. For a continuous function $f$ on an open domain $U$ in $\mathcal{A}_{\Lambda}$ there exists a generalized operator $\hat{f}$ in the sense of distributions on rectifiable paths in $U$. Then Definition 1 has the natural extension on $\mathcal{A}_{\Lambda}$.

In Note 2.8 it can be used $l_{2}(\mathbf{R})^{m}$ instead of $\mathbf{R}^{2^{\mathbf{r}} \mathbf{m}}$ and represent the differential forms $\eta$ over $\mathcal{A}_{\infty}$ as the pointwise limits (or to use the uniform converegence on compact subsets) of differential forms over $\mathcal{A}_{r}$ for $r$ tending to the infinity, since $z_{r} \rightarrow z$ while $r$ tends to the infinity, where $z \in \mathcal{A}_{\infty}, z_{r}:=P_{r}(z)$. In the case of $\mathcal{A}_{\Lambda}$ when $\operatorname{card}(\Lambda)>\aleph_{0}$ this can be used pointwise, since for each $z \in \mathcal{A}_{\Lambda}$ there exists a subalgebra isomorphic with $\mathcal{A}_{\infty}$ and containing $z$. In the general case: 
(i) $\eta(z, \tilde{z})=\sum_{I, J} \eta_{I, J}\left\{\left(d^{p_{1}} z^{\wedge I_{1}} \alpha_{1} \wedge \ldots \wedge d^{p_{n}} z^{\wedge I_{n}} \alpha_{n} \wedge d^{t_{1}} \tilde{z}^{\wedge J_{1}} \beta_{1} \wedge \ldots \wedge d^{t_{n}} \tilde{z}^{\wedge J_{n}} \beta_{n}\right\}_{q(|I|+|J|+2 n)}\right.$ this is the differential form over $\mathcal{A}_{\Lambda}$, where each $\eta_{I, J}(z, \tilde{z})$ is a continuous function on an open subset $U_{n}$ in $\mathcal{A}_{\Lambda}^{n}$ with values in $\mathcal{A}_{\Lambda}, I=\left(I_{1}, \ldots, I_{n}\right), J=\left(J_{1}, \ldots, J_{n}\right),|I|:=I_{1}+\ldots+I_{n}$, $1 \leq p_{1} \leq p_{2} \leq \ldots \leq p_{n} \in \mathbf{N}, 1 \leq t_{1} \leq t_{2} \leq \ldots \leq t_{n} \in \mathbf{N}, 0 \leq I_{k} \in \mathbf{Z}, 0 \leq J_{k} \in \mathbf{Z}, \alpha_{k}, \beta_{k} \in \mathcal{A}_{\Lambda}$ are constants for each $k=1, \ldots, n, d^{p} z^{0}:=1, d^{p} \tilde{z}^{0}:=1, n \in \mathbf{N}, \pi_{n}^{l}\left(U_{l}\right) \subset U_{n}$ for all $l \geq n$, where $\pi_{n}^{l}: \mathcal{A}_{\Lambda}^{l} \rightarrow \mathcal{A}_{\Lambda}^{n}$ is the natural projection for each $l \geq n$. The converegence on the right side of Formula $(i)$ in the case of an infinite series by $I$ or $J$ is supposed relative to $C_{b}^{0}\left(W, \mathcal{A}_{\Lambda}^{\wedge *}\right)$-topology of the uniform convergence on $W$, where $W=p r-\lim \left\{U_{n}, \pi_{n}^{l}, \mathbf{N}\right\}$, $\mathcal{A}_{\Lambda}^{\wedge *}$ is supplied with the norm topology inherited from the topologically adjoint space of all poly $\mathbf{R}$-homogeneous $\mathcal{A}_{\Lambda}$-additive functionals.

In Note 2.10 [20] define $\mathcal{A}_{\Lambda, s, p}$ an use projections $\pi_{s, p, t}$ for each $s \neq p \in \mathbf{b}$. Theorems 2.11, 2.15 and Corollaries 2.13, 2.15.1 are transferrable on $\mathcal{A}_{\Lambda}$ with $\operatorname{card}(\Lambda) \geq \aleph_{0}$ by imposing the condition of $\left(2^{r}-1\right)$-connectedness $P_{r}(U)=: U_{r}$ for each $r \geq 3$ and every embedding of $\mathcal{A}_{\infty}$ into $\mathcal{A}_{\Lambda}$ for $\operatorname{card}(\Lambda)>\aleph_{0}$ while corresponding $\mathcal{A}_{r} \subset \mathcal{A}_{\infty}$ considering $\pi_{s, p, t}(U)$ for each $s=i_{2 k}, p=i_{2 k+1}, 0 \leq k \in \mathbf{Z}$. Then Definitions 2.12,2.14 and Theorem 2.16, Notes 2.17,3.1 are also accomplished for $\mathcal{A}_{\Lambda}$. Corollary 3.3 in this case follows from Theorem 3.6.2 [20].

As for $\mathcal{A}_{\infty}$ the algebra $\mathcal{A}_{\Lambda}$ with $\operatorname{card}(\Lambda)>\aleph_{0}$ has not any finite and even countable set of constants $\left\{a_{s}, b_{s}\right\}$ in $\mathcal{A}_{\Lambda}$ such that $z^{*}=\sum_{s} a_{s} z b_{s}$ for each $z \in \mathcal{A}_{\Lambda}$ could be written in as such series or sum. That is the algebraic antiautomorphism of order two $\theta(z):=z^{*}$ with $\theta \circ \theta=i d$ is not internal in $\mathcal{A}_{\Lambda}$ and indeed $z$ and $z^{*}$ are algebraically independent variables in such infinite dimensional Cayley-Dickson algebra.

2.2.5. Proposition. Let $U$ be an open subset in $\mathcal{A}_{p}$ and $f: U \rightarrow \mathcal{A}_{p}$ be a function on $U$, where $2 \leq p \leq \infty$, let also $\mathcal{A}_{\Lambda}$ be the Cayley-Dickson algebra as in §2.2.2 with card $(\Lambda) \geq \aleph_{0}$, then $f$ is z-superdifferentiable if and only if there exists an open subset $W$ in $\mathcal{A}_{\Lambda}$ and a $z$-superdifferentiable function $g: W \rightarrow \mathcal{A}_{\Lambda}$ such that its restriction on $U$ coincides with $f$, $\left.g\right|_{U}=f$.

Proof. In view of Theorem 2.2.3 there exists the embedding of $\mathcal{A}_{p}$ into $\mathcal{A}_{\Lambda}$. If $g$ is $z$ superdifferentiable on $W$, then from the definition it follows, that $\left.g\right|_{V}$ is $z$-superdifferentiable, where $V=W \cap \mathcal{A}_{p}$ and $V$ is open in $\mathcal{A}_{p}$. Vice versa if $f$ is $z$-superdifferentiable on $U$, then it is locally $z$-analytic on $U$ (see Theorems 2.15 and $3.10[20]$ ). For each $z_{0} \in U$ there exists a power series in $\left(z-z_{0}\right)$ converging in a ball $B\left(\mathcal{A}_{p}, z_{0}, r\right)$ with the centre $z_{0}$ and positive radius $r>0$ the expansion coefficients of which belong to $\mathcal{A}_{p}$. Therefore, in the variable $\left(z-z_{0}\right)$ this series uniformly converges in $B\left(\mathcal{A}_{\Lambda}, z_{0}, r^{\prime}\right)$ for each $0<r^{\prime}<r$. The union of such balls $B\left(\mathcal{A}_{\Lambda}, z_{0}, r^{\prime}\right)$ is the open subset in $\mathcal{A}_{\Lambda}$ which we denote by $W$. On each open intersection of each corresponding pair of such balls the functions given by such series coincide, that gives the $z$-superdifferentiable function $g$ on $W$ with $\left.g\right|_{U}=f$. Certainly there can be found others $z$-superdifferentiable extensions $g$ of $f$.

2.3. Theorem. Let $U$ be a bounded open subset in $\mathcal{A}_{\Lambda}$ and let $f: U \rightarrow \mathcal{A}_{\Lambda}$ be a bounded continuous function. Then there exists a continuous function $u(z)$ which is a solution of the equation

$$
\text { (1) }(\partial u(z) / \partial \tilde{z})=\hat{f}
$$

in $U$, in particular, $(\partial u(z) / \partial \tilde{z}) .1=f(z)$.

Proof. Using embeddings of $\mathcal{A}_{p}, 2 \leq p \in \mathbf{N}$, into $\mathcal{A}_{\Lambda}$ it is sufficient to prove this statement for arbitrary $2 \leq p \in \mathbf{N}$. Take $2 \leq p \in \mathbf{N}$ and $\mathcal{A}_{p}$ one-forms $d \zeta_{l}$ expressible through $d \zeta$ as $\sum_{j=1}^{k(l)} P_{j, 1, l} d \zeta P_{j, 2, l}$ with fixed nonzero $P_{j, q, l} \in \mathcal{A}_{p}$, where $l=1,2,3, \ldots, 2^{p}, k(l) \in \mathbf{N}$. Choose $d \zeta_{l}$ to be satisfying conditions $\left.d \zeta_{2^{p}} \wedge \nu=\xi(z)\left(\ldots\left(\left(d^{1} z \wedge d^{2} z\right) \wedge d^{3} z\right) \ldots\right) \wedge d^{2^{p}} z\right)$, $d \tilde{\zeta}_{2^{p}} \wedge \nu=0$, where

$$
\left.\left.\left.\left.\left.\nu=\left(\partial_{\zeta_{1}} \operatorname{Ln}\left(\zeta_{1}-\zeta_{2}\right)\right) M_{1}^{*}\right]\right) .\left[\left(\partial_{\zeta_{2}} \operatorname{Ln}\left(\zeta_{2}-\zeta_{3}\right)\right) M_{2}^{*}\right]\right) \ldots\right) .\left[\left(\partial_{\zeta_{m}} \operatorname{Ln}\left(\zeta_{m}-z\right)\right) M_{m}^{*}\right]\right),
$$


$m=2^{p}-1$ as in $\S 2.1, z=\sum_{l=1}^{2^{p}}{ }^{l} z i_{l-1} \in U,{ }^{l} z \in \mathbf{R}, i_{0}, \ldots, i_{2^{p-1}}$ are generators of $\mathcal{A}_{p}$, $\xi: U \rightarrow \hat{\mathcal{A}}_{p}$ is a function nonzero and finite almost everywhere on $U$ relative to the Lebesgue measure. Then there exist $d \zeta_{2^{p}}$ and $\nu$ such that the continuous function

$$
u(z):=-(2 \pi)^{1-2^{p}} \int_{U}\left(\hat{f}\left(\zeta_{1}\right) \cdot d \tilde{\zeta}_{2^{p}}\right) \wedge \nu
$$

is a solution of equation (1). To demonstrate this take closed curves (paths) $\gamma_{j}$ in $U$ as in $\S 2.1$ and $\S 2.2 .4$, for example, such that $\zeta_{j} \in \gamma_{j}$ satisfy conditions: $\left(\zeta_{2 s-1}-\zeta_{2 s}\right)=\eta_{2 s-1}$ for each $s=1, \ldots, 2^{p-1}-1, \zeta_{2^{p}-1}=-r \eta_{2^{p}-1}^{*}$ with $0<r<1$, where $\eta_{2 s-1}$ and $\eta_{2 s}$ belong to the plane $i_{2 s-1} \mathbf{R} \oplus i_{2 s} \mathbf{R}, \eta_{2 s}=\tilde{\eta}_{2^{s}-1}$ for each $s=1, \ldots, 2^{p-1}, \tilde{\eta}_{1}=\eta_{2^{p}}$. Hence $d \eta_{1} \wedge d \eta_{1}=0$, $d \eta_{2} \wedge d \eta_{3}^{*}=0, \ldots, d \eta_{2^{p}-1} \wedge d \eta_{2^{p}}^{*}=0, d \eta_{2^{p}} \wedge d \eta_{2^{p}}=0$,

(i) $\eta_{v}^{k} d \eta_{v}=\left(d \eta_{v}\right) \eta_{v}^{k}$ for each $v$ for $k=1$ and $k=-1$.

These variables are expressible as $\zeta_{l}=\sum_{j=1}^{k(l)} P_{j, 1, l} \zeta P_{j, 2, l}$ (see $\S \S 3.7$ and 3.28 [19]). Therefore, there exists a subgroup of the group of all $\mathcal{A}_{p}$-holomorphic diffeomorphisms of $U$ preserving Conditions $(i i)$ and the construction given above has natural generalizations with the help of such diffeomorphisms.

Supoose at first, that $f$ is continuously differentiable in $U$. Each $\zeta_{j}$ is expressible in the form $\zeta_{j}=\sum_{l}{ }^{l} b_{j} S_{l}$, where ${ }^{l} b_{j} \in \mathbf{R}$ are real variables, $S_{l} \in\left\{i_{0}, i_{1}, \ldots, i_{2^{p}-1}\right\}$, hence differentials $\left(\partial f / \partial \zeta_{j}\right) \cdot d \zeta_{j}=\sum_{l}\left\{(\partial f / \partial z) . S_{l} d^{l} b_{j}+(\partial f / \partial \tilde{z}) \cdot \tilde{S}_{l} d^{l} b_{j}\right\}$ are defined. Consider a fixed $z_{0} \in U$. We take a $C^{\infty}$-function $\chi$ on $\mathcal{A}_{p}$ such that $\chi=1$ in a neighbourhood $V$ of $z_{0}, V \subset U, \chi=0$ in a neighbourhood of $\mathcal{A}_{p} \backslash U$. Then $u=u_{1}+u_{2}$, where

$$
\begin{gathered}
u_{1}(z):=-(2 \pi)^{1-2^{p}} \int_{U}\left[\chi\left(\zeta_{1}\right)\left(\hat{f}\left(\zeta_{1}\right) \cdot d \tilde{\zeta}_{2^{p}}\right)\right] \wedge \nu \\
u_{2}(z):=-(2 \pi)^{1-2^{p}} \int_{U}\left[\left(1-\chi\left(\zeta_{1}\right)\right)\left(\hat{f}\left(\zeta_{1}\right) \cdot d \tilde{\zeta}_{2^{p}}\right)\right] \wedge \nu .
\end{gathered}
$$

Then

$$
\begin{gathered}
u(z):=-(2 \pi)^{1-2^{p}} \int_{\mathcal{A}_{p}}\left[\chi\left(\zeta_{1}+z\right)\left(\hat{f}\left(\zeta_{1}+z\right) \cdot d \tilde{\zeta}_{2^{p}}\right)\right] \wedge \psi, \text { where } \\
\left.\left.\left.\left.\left.\psi:=\left(\partial_{\zeta_{1}} \operatorname{Ln}\left(\zeta_{1}-\zeta_{2}\right)\right) M_{1}^{*}\right]\right) \cdot\left[\left(\partial_{\zeta_{2}} \operatorname{Ln}\left(\zeta_{2}-\zeta_{3}\right)\right) M_{2}^{*}\right]\right) \ldots\right) \cdot\left[\left(\partial_{\zeta_{m}} \operatorname{Ln}\left(\zeta_{m}\right)\right) M_{m}^{*}\right]\right) .
\end{gathered}
$$

Since $\partial_{\zeta_{2^{p}}}\left\{\left[\chi\left(\zeta_{1}+z\right) \hat{f}\left(\zeta_{1}+z\right) \cdot\right] \wedge \psi\right\}=0$ and $\left(\partial / \partial \tilde{\zeta}_{2^{p}}\right)\left\{\left[\chi\left(\zeta_{1}+z\right) \hat{f}\left(\zeta_{1}+z\right) \cdot\right] \wedge \psi\right\} \cdot d \tilde{\zeta}_{2^{p}}=$ $\partial_{\tilde{\zeta}_{2 p}}\left\{\left[\chi\left(\zeta_{1}+z\right) \hat{f}\left(\zeta_{1}+z\right).\right] \wedge \psi\right\}$, then due to Equations $(i, i i)$ $(\partial u(z) / \partial \tilde{z})=-(2 \pi)^{1-2^{p}} \int_{\mathcal{A}_{p}} \partial_{\tilde{\zeta}_{2 p}}\left\{\left[\chi\left(\zeta_{1}+z\right) \hat{f}\left(\zeta_{1}+z\right)\right] \wedge \psi\right\}$.

In view of Theorem 2.1 applied to $\hat{f} . S$ for each $S \in\left\{i_{0}, i_{1}, \ldots, i_{2^{p}-1}\right\}$ we have $\left(\partial u_{1} / \partial \tilde{z}\right)=\hat{f}$ in $V$, consequently, $(\partial u / \partial \tilde{z})=\hat{f}$ in a neighbourhood of $z_{0}$.

Taking a sequence $f^{n}$ of continuously differentiable functions uniformly converging to $f$ on $U$ we get the corresponding $u^{n}$ such that in the sence of distributions $(\partial u / \partial \tilde{z})=$ $\lim _{n \rightarrow \infty}\left(\partial u^{n} / \partial \tilde{z}\right)=\lim _{n} \hat{f}^{n}=\hat{f}$. Consider the family of all embeddings $\theta$ of $\mathcal{A}_{p}$ to $\mathcal{A}_{\Lambda}$, $2 \leq p<\infty$. There exists the generalized function (operator) $\hat{f}$ on $U$, hence there exists the restriction $\hat{f}_{p, \theta}$ on $U \cap \theta\left(\mathcal{A}_{p}\right)$ for each $(p, \theta)$. As the function this restriction evidently exists. For distributions it is possible to take them on a base space of cylindrical functions on the algebra of cylindrical subsets with bases in the projection $\theta\left(\mathcal{A}_{p}\right)$. Each rectifiable path $\gamma$ is the limit of the uniformly converging net of paths $\gamma_{p, \theta}$, since $\gamma([0,1])$ is compact. Therefore, such restriction exists in the sence of distributions.

Thus there exists the solution $u_{p, \theta}$ of (1) given by (2) on $U \cap \theta\left(\mathcal{A}_{p}\right)$. The family of all $(p, \theta)$ is directed: $\left(p_{1}, \theta_{1}\right) \leq\left(p_{2}, \theta_{2}\right)$ if and only if $p_{1} \leq p_{2}$ and $\theta_{1}\left(\mathcal{A}_{p_{1}}\right) \subset \theta_{2}\left(\mathcal{A}_{p_{2}}\right)$. Since $\hat{f}_{p, \theta}$ 
converges to $\hat{f}$ in the sense of distributions by the ultrafilter of the set $\{(p, \theta)\}$, then $u_{p, \theta}$ converges to the solution $u$ on $U$, since there exists $\partial u(z) / \partial \tilde{z}=\hat{f}(z)$.

2.4. Theorem. Let $U$ be an open subset in $\mathcal{A}_{p}^{n}, 2 \leq p \in \mathbf{N}, n \in \mathbf{N}$. Then for every compact subset $K$ in $U$ and every multi-order $k=\left(k_{1}, \ldots, k_{n}\right)$, there exists a constant $C>0$ such that

$$
\max _{z \in K}\left|\partial^{k} f(z)\right| \leq C \int_{U}|f(z)| d \sigma_{2^{p} n}
$$

for each $\mathcal{A}_{p}$-holomorphic function $f$, where $d \sigma_{2^{p} n}$ is the Lebesgue measure in $\mathcal{A}_{p}^{n}$.

2.5. Corollary. Let $U$ be an open subset in $\mathcal{A}_{p}^{n}, 2 \leq p \in \mathbf{N}, n \in \mathbf{N}$, and let $f_{l}$ be a sequence of $\mathcal{A}_{p}$-holomorphic functions in $U$ which is uniformly bounded on every compact subset of $U$. Then there is a subsequence $f_{k_{j}}$ converging uniformly on every compact subset of $U$ to a limit in $C_{z}^{\omega}\left(U, \mathcal{A}_{p}\right)$.

Proofs of Theorem 2.4 and Corollary 2.5 follow from Theorem 2.1 above and Theorem 3.9 [19] (see also [20]) analogously to Theorem 1.1.13 and Corollary 1.1.14 [7].

2.6. Definitions. Let $U$ be an open subset in $\mathcal{A}_{p}^{n}$ and $f: U \rightarrow \mathcal{A}_{p}^{m}$ be an $\mathcal{A}_{p}$-holomorphic function, then the matrix: $J_{f}(z):=\left(\partial f_{j}(z) / \partial z_{k}\right)$ is called the $\mathcal{A}_{p^{-}}$Jacobi matrix, where $j=1, \ldots, m, k=1, \ldots, n$. To this operator matrix there corresponds a real $\left(2^{p} m\right) \times\left(2^{p} n\right)$ matrix while $2 \leq p \in \mathbf{N}$ or operator from $X^{m}$ into $X^{n}$ of the underlying real Hilbert space $X$ of $\mathcal{A}_{p}$ for infinite $p=\Lambda$. Denote by $\operatorname{rank}_{\mathbf{R}}\left(J_{f}(z)\right)$ a rank of a real matrix or operator corresponding to $J_{f}(z)$. This rank may be infinite. Then $f$ is called regular at $z \in U$, if $\operatorname{rank}_{\mathbf{R}}\left(J_{f}(z)\right)=2^{p} \min (n, m)$ for finite $p$ or $\operatorname{ker}\left(f^{\prime}(z)\right)=0$ and $\operatorname{Range}\left(f^{\prime}(z)\right)$ is algebraically isomorphic with $\mathcal{A}_{\Lambda}^{m}$ such that $\operatorname{Range}\left(f^{\prime}(z)\right) \oplus \mathcal{A}_{\Lambda}^{n-m}=\mathcal{A}_{\Lambda}^{n}$ when $m \leq n$ or Range $\left(f^{\prime}(z)\right)=X^{n}$ and $\operatorname{ker}\left(f^{\prime}(z)\right)$ is algebraically isomorphic with $\mathcal{A}_{\Lambda}^{m-n}$ while $m>n$. If $U$ and $V$ are two open subsets in $\mathcal{A}_{p}^{n}$, then a bijective surjective mapping $f: U \rightarrow V$ is called $\mathcal{A}_{p}$-biholomorphic if $f$ and $f^{-1}: V \rightarrow U$ are $\mathcal{A}_{p}$-holomorphic.

2.7. Proposition. Let $U$ and $V$ be open subsets in $\mathcal{A}_{p}^{n}$ and $\mathcal{A}_{p}^{m}$ respectively. If $f: U \rightarrow$ $\mathcal{A}_{p}^{m}$ and $g: V \rightarrow \mathcal{A}_{p}^{k}$ are $\mathcal{A}_{p}$-holomorphic functions such that $f(U) \subset V$, then $g \circ f: U \rightarrow \mathcal{A}_{p}^{k}$ is $\mathcal{A}_{p}$-holomorphic and $J_{g \circ f}(z)=J_{g}(f(z)) .\left(J_{f}(z) . h\right)$ for each $h \in \mathcal{A}_{p}^{n}$.

Proof. In view of Definition 2.2 and Theorems 2.15 and $3.10[20]\left(\partial g_{j}(f(z)) / \partial z_{l}\right) \cdot \zeta=$ $\left.\sum_{s=1}^{m} \sum_{l=1}^{k}\left(\partial g_{j}(\xi) / \partial \xi_{s}\right)\right|_{\xi=f(z)} \cdot\left(\partial f_{s}(z) / \partial z_{l}\right) . h_{l}$, where $h=\left(h_{1}, \ldots, h_{n}\right), h_{l} \in \mathcal{A}_{p}$ for each $l=$ $1, \ldots, n$, since $f(U) \subset V$ and this is evident for $\mathcal{A}_{p}$-polynomial functions and hence for locally converging series of $\mathcal{A}_{p}$-holomorphic functions.

2.8. Proposition. Let $U$ be a neighbourhood of $z \in \mathcal{A}_{p}^{n}$ and let $f: U \rightarrow \mathcal{A}_{p}^{n}$ be an $\mathcal{A}_{p}$-holomorphic function. Then $f$ is $\mathcal{A}_{p}$-biholomorphic in some neighbourhood $W$ of $z$ if and only if $f$ is regular at a point $z \in U$.

Proof. From Proposition 2.7 it follows, that the condition of regularity of $f$ on $U$ is necessary. Prove the sufficiency. In view of Definition 2.2, Theorems 2.15 and 3.10 and Note $3.11[20]$ an incerement of $f$ can be written in the form $f(z+\zeta)=f(z)+f^{\prime}(z) \cdot \zeta+O\left(|\zeta|^{2}\right)$ for each $\zeta \in \mathcal{A}_{p}^{n}$ such that $z+\zeta \in U$. Then there exists a neighborhood $W \supset B\left(z, 2 \epsilon, \mathcal{A}_{p}^{n}\right)$ in which $|g(z+\zeta)| \leq C|\zeta|^{2}$, where $0<\epsilon<(2 C)^{-1}, C$ is a positive constant, $g:=i d-f$. Thus there exists an $\mathcal{A}_{p}$-holomorphic function $w$ on an open neighbourhood $W$ of $z$ in $U$ such that $w$ is given by the series $w=\sum_{k=1}^{\infty} g_{k}$, where $g_{k+1}=g \circ g_{k}$ for each $k \in \mathbf{N}$ and $g_{1}:=g, g:=i d-f$, since for each $\eta \in W$ there exists $r>0$ such that $B\left(\eta, r, \mathcal{A}_{p}\right) \subset U$ and the series for $w$ is convergent on $B\left(\eta, r, \mathcal{A}_{p}\right)$ with $w\left(B\left(z, \epsilon, \mathcal{A}_{p}^{n}\right)\right) \subset B\left(z, 2 \epsilon, \mathcal{A}_{p}^{n}\right)$.

Since $f^{\prime}(z)$ is the continuous epimorphism from $\mathcal{A}_{p}^{n}$ onto $\mathcal{A}_{p}^{n}$, then its graph is closed. On the other hand, $f^{\prime}(z)$ is bijective and there exists the $\mathbf{R}$-linear operator $\left(f^{\prime}(z)\right)^{-1}$. The graph of it $\operatorname{Gr}\left(f^{\prime}(z)\right)^{-1}=\left\{(x, y): x=f^{\prime}(z) . y ; x, y \in \mathcal{A}_{p}^{n}\right\}$ is closed in $\mathcal{A}_{p}^{n} \otimes \mathcal{A}_{p}^{n}$, since the graph of $f^{\prime}(z)$ is closed. In view of the closed mapping theorem (see 14.3.4 [23]) $\left(f^{\prime}(z)\right)^{-1}$ is continuous. Thus the operator $f^{\prime}(z)$ is invertible. In view of the inverse mapping theorem 
(see $\S$ X.7 [27]) there exists $f^{-1}$ continuusly (Frechét) differentiable on a neighborhood $W$ of $f(z)$. Since $\partial f(z) / \partial \tilde{z}=0$, then $\partial f^{-1}(\zeta) / \partial \tilde{\zeta}=0$ on $W$.

For $\eta$ in a sufficiently small neighborhood $W$ of $z$ there is satisfied the inequality $\| 1-$ $f^{\prime}(z)^{-1} f^{\prime}(\eta) \|<1$, consequently, $f^{\prime}(\eta)$ is invertible for each $\eta \in W$. The operator $f^{\prime}(\eta)$ is continuous by $\eta$ on $U$, hence there exists a neighbourhood $V$ of $z$ such that $f$ is regular on $V$, since $f^{\prime}(\eta)$ is $\mathbf{R}$-homogeneous and $\mathcal{A}_{p}$-additive and $f^{\prime}(z)\left(\mathcal{A}_{p}^{n}\right)=\mathcal{A}_{p}^{n}$. Hence $f(V)$ is open in $\mathcal{A}_{p}^{n}$. Since $w$ is the limit of the uniformly convergent series of $\mathcal{A}_{p}$-holomorphic functions, then $w$ is $\mathcal{A}_{p}$-holomorphic on $W$. From $(i d+h) \circ f=f \circ(i d+h)=i d$ on $B\left(z, \epsilon, \mathcal{A}_{p}^{n}\right)$ it follows, that $f$ is $\mathcal{A}_{p}$-biholomorphic on a neighbourhood of $z$.

2.9. Corollary. Let $X$ be a subset in $\mathcal{A}_{p}^{n}, 2 \leq p<\infty$ or $p=\Lambda$, and $k \in\{1,2, \ldots, n-1\}$, then the following conditions are equivalent:

(i) for each $\zeta \in X$ there exists an $\mathcal{A}_{p}$-biholomorphic map $f=\left(f_{1}, \ldots, f_{n}\right)$ in some neighbourhood $U$ of $\zeta$ such that $f$ is regular on $U$ and $X \cap U=\left\{z \in U: f_{k+1}(z)=0, \ldots, f_{n}(z)=0\right\}$;

(ii) for each $\zeta \in X$ there exists a neighbourhood $V$ of $\zeta$ and a regular $\mathcal{A}_{p}$-holomorphic map $g: V \rightarrow \mathcal{A}_{p}^{n-k}$ such that $X \cap V=\{z \in V: g(z)=0\}$.

Proof. The implication $(i) \Rightarrow(i i)$ follows by taking $g=\left(f_{k+1}, \ldots, f_{n}\right)$ on $V=U$. To prove implication $(i i) \Rightarrow(i)$ take $\zeta \in X, g$ and $V$ as in (ii). There exists the $\mathbf{R}$-linear operator $G^{\prime}$ corresponding to $g^{\prime}(\zeta)$ from $\mathcal{A}_{p}^{n}$ onto $\mathcal{A}_{p}^{n-k}$. Thus there exists a right $\mathcal{A}_{p}$-superlinear operator $P$ from $\mathcal{A}_{p}^{n}$ onto $\mathcal{A}_{p}^{k}$ such that $P \oplus g^{\prime}(\zeta)$ from $\mathcal{A}_{p}^{n}$ onto $\mathcal{A}_{p}^{n}$ is invertible. The graph of it $\operatorname{Gr}\left(\left(P \oplus g^{\prime}(\zeta)\right)^{-1}=\left\{(x, y): x=\left(P \oplus g^{\prime}(\zeta)\right)^{-1} . y ; x, y \in \mathcal{A}_{p}^{n}\right\}\right.$ is closed in $\mathcal{A}_{p}^{n} \otimes \mathcal{A}_{p}^{n}$, since the graph of $\left(P \oplus g^{\prime}(\zeta)\right)$ is closed. In view of the closed mapping theorem (see 14.3.4 [23]) $\left(P \oplus g^{\prime}(\zeta)\right)^{-1}$ is continuous. Thus the operator $\left(P \oplus g^{\prime}(\zeta)\right)$ is invertible. In view of the implicit mapping theorem and addition 3 to it (see $\S$ X.7 $[27])$ there exists $(P \oplus g)^{-1}$ continuusly (Frechét) differentiable on a neighborhood $W$ of $(P \oplus g)(\zeta)$.

Put $f(z)=(P z, g(z))$ for $z \in V$. By Theorem $2.8 f$ is $\mathcal{A}_{p}^{n}$-biholomorphic in some neighborhood $U \subset V$ of $\zeta$. Then $X \cap U=\left\{z \in U: f_{k+1}(z)=0, \ldots, f_{n}(z)=0\right\}$, since $\left(f_{k+1}, \ldots, f_{n}\right)=g$ and $X \cap U=\{z \in U: g(z)=0\}$.

2.10. Definitions. Let $U$ be an open subset in $\mathcal{A}_{p}^{n}, 2 \leq p<\infty$ or $p=\Lambda$. A subset $X$ in $U$ is called a $\mathcal{A}_{p}$-submanifold of $\mathcal{A}_{p}^{n}$ if the equivalent conditions of Corollary 2.9 are satisfied. If in addition $X$ is a closed subset in $U$, then $X$ is called a closed $\mathcal{A}_{p}$-submanifold of $U$. This definition is the particular case of the following general definition.

An $\mathcal{A}_{p}$-holomorphic manifold of $\mathcal{A}_{p}$-dimension $n$ is a real $2^{p} n$-dimensional or $\operatorname{card}(\Lambda)$ dimensional $C^{\infty}$-manifold $X$ together with a family $\left\{\left(U_{j}, \phi_{j}\right): j \in \Psi\right\}$ of charts such that

(i) each $U_{j}$ is an open subset in $X$ and $\bigcup_{j \in \Psi} U_{j}=X$, where $\Psi$ is a set;

(ii) for each $j \in \Psi$ a mapping $\phi_{j}: U_{j} \rightarrow V_{j}$ is a homeomorphism on an open subset $V_{j}$ in $\mathcal{A}_{p}^{n}$

(iii) for each $j, l \in \Psi$ a connection mapping $\phi_{j} \circ \phi_{l}^{-1}$ is an $\mathcal{A}_{p}$-biholomorphic map (see $\left.\S 2.6\right)$ from $\phi_{l}\left(U_{j} \cap U_{l}\right)$ onto $\phi_{j}\left(U_{j} \cap U_{l}\right)$. Such system is called an $\mathcal{A}_{p^{-}}$-holomorphic atlas $\operatorname{At}(X):=$ $\left\{\left(U_{j}, \phi_{j}\right): j \in \Psi\right\}$. Each chart $\left(U_{j}, \phi_{j}\right)$ provides a system of $\mathcal{A}_{p}$-holomorphic coordinates induced from $\mathcal{A}_{p}^{n}$. For short we shall write $\mathcal{A}_{p}$-manifold instead of $\mathcal{A}_{p}$-holomorphic manifold and $\mathcal{A}_{p}$-atlas instead of $\mathcal{A}_{p}$-holomorphic atlas if other will not be specified.

For two $\mathcal{A}_{p}$-manifolds $X$ and $Y$ with atlases $\operatorname{At}(X):=\left\{\left(U_{j}, \phi_{j}\right): j \in \Psi_{X}\right\}$ and $\operatorname{At}(Y):=$ $\left\{\left(W_{l}, \psi_{l}\right): l \in \Psi_{Y}\right\}$ a function $f: X \rightarrow Y$ is called $\mathcal{A}_{p^{-}}$holomorphic if $\psi_{l} \circ f \circ \phi_{j}^{-1}$ is $\mathcal{A}_{p^{-}}$ holomorphic on $\phi_{j}\left(U_{j} \cap f^{-1}\left(W_{l}\right)\right)$. If $f: X \rightarrow Y$ is an $\mathcal{A}_{p}$-biholomorphic epimorphism, then $X$ and $Y$ are called $\mathcal{A}_{p}$-biholomorphically equivalent.

A subset $Z$ of an $\mathcal{A}_{p^{-}}$-manifold $X$ is called an $\mathcal{A}_{p^{-}}$submanifold, if $\phi_{j}\left(U_{j} \cap Z\right)$ is an $\mathcal{A}_{p^{-}}$ submanifold in $\mathcal{A}_{p}^{n}$ for each chart $\left(U_{j}, \phi_{j}\right)$. If additionally $Z$ is closed in $X$, then $Z$ is called a closed $\mathcal{A}_{p}$-submanifold. 
2.11. Theorem. Let $n \geq 2, f_{1}, \ldots, f_{n} \in C_{0,(z, \tilde{z})}^{1}\left(\mathcal{A}_{p}^{n}, \mathcal{A}_{p}\right)$ with $2 \leq p<\infty$ or $p=\Lambda$ be a family of continuously $\mathcal{A}_{p}(z, \tilde{z})$-superdifferentiable functions satisfying compatibility conditions:

$$
\text { (i) } \partial f_{j} / \partial \tilde{z}_{k}=\partial f_{k} / \partial \tilde{z}_{j} \text { for each } j, k=1, \ldots, n,
$$

where $C_{0,(z, \tilde{z})}^{1}\left(\mathcal{A}_{p}^{n}, \mathcal{A}_{p}\right)$ is the subspace of $C_{(z, \tilde{z})}^{1}\left(\mathcal{A}_{p}^{n}, \mathcal{A}_{p}\right)$ of functions with closed bounded support. Then there exists $u \in C_{0,(z, \tilde{z})}^{1}\left(\mathcal{A}_{p}^{n}, \mathcal{A}_{p}\right)$ satisfying the following $\tilde{\partial}$-equation:

$$
\text { (ii) } \partial u / \partial \tilde{z}_{j}=\hat{f}_{j}, \quad j=1, \ldots, n \text {; }
$$

in particular, $\left(\partial u / \partial \tilde{z}_{j}\right) .1=f_{j}$.

Proof. Using the beginning and the end of the proof of Theorem 2.3 we reduce the proof of Theorem 2.11 to the case of finite $p$ mentioning, that the intersection $A \cap \theta\left(\mathcal{A}_{p}\right)^{n}$ of a closed bounded subset $A$ in $\mathcal{A}_{\Lambda}^{n}$ for finite $p$ is compact. We put

$$
\begin{gathered}
(i i i) \quad u(z):=-(2 \pi)^{1-2^{p}} \int_{\theta\left(\mathcal{A}_{p}\right)}\left[\left(\hat{f}_{1}\left(\zeta_{1}, z_{2}, \ldots, z_{n}\right) \cdot d \tilde{\zeta}_{2^{p}}\right) \wedge \eta\right], \text { where } \\
\left.\left.\left.\left.\left.\eta:=\left(\partial_{\zeta_{1}} \operatorname{Ln}\left(\zeta_{1}-\zeta_{2}\right)\right) M_{1}^{*}\right]\right) \cdot\left[\left(\partial_{\zeta_{2}} \operatorname{Ln}\left(\zeta_{2}-\zeta_{3}\right)\right) M_{2}^{*}\right]\right) \ldots\right) \cdot\left[\left(\partial_{\zeta_{m}} \operatorname{Ln}\left(\zeta_{m}-z\right)\right) M_{m}^{*}\right]\right),
\end{gathered}
$$

$m=2^{p}-1$ (see $\left.\S 2.3\right)$. By changing of variables we get

$$
\begin{gathered}
u(z):=-(2 \pi)^{1-2^{p}} \int_{\mathcal{A}_{p}}\left[\left(\hat{f}_{1}\left(z_{1}+\zeta_{1}, z_{2}, \ldots, z_{n}\right) \cdot d \tilde{\zeta}_{2^{p}}\right) \wedge \psi\right], \text { where } \\
\left.\left.\left.\left.\left.\psi:=\left(\partial_{\zeta_{1}} \operatorname{Ln}\left(\zeta_{1}-\zeta_{2}\right)\right) M_{1}^{*}\right]\right) \cdot\left[\left(\partial_{\zeta_{2}} \operatorname{Ln}\left(\zeta_{2}-\zeta_{3}\right)\right) M_{2}^{*}\right]\right) \ldots\right) \cdot\left[\left(\partial_{\zeta_{m}} \operatorname{Ln}\left(\zeta_{m}\right)\right) M_{m}^{*}\right]\right),
\end{gathered}
$$

$m=2^{p}-1$. Therefore, $u \in C_{(z, \tilde{z})}^{1}\left(\mathcal{A}_{p}^{n}, \mathcal{A}_{p}\right)$. Due to Theorem $2.3 \partial u / \partial \tilde{z}_{1}=\hat{f}_{1}$ in $\mathcal{A}_{p}^{n}$. In view of Theorem 2.1 and the condition $\partial f_{1} / \partial \tilde{z}_{k}=\partial f_{k} / \partial \tilde{z}_{1}$ the following equality is satisfied

$$
\hat{f}_{k}(z)=-(2 \pi)^{1-2^{p}} \int_{\mathcal{A}_{p}}\left\{\left[\partial \hat{f}_{k}\left(\zeta_{1}, z_{2}, \ldots, z_{n}\right) / \partial \tilde{\zeta}_{1}\right] \cdot d \tilde{\zeta}_{2^{p}}\right\} \wedge \psi
$$

hence $\partial u / \partial \tilde{z}_{k}=\hat{f}_{k}$ for $k=2, \ldots, n$, that is, $u$ satisfies equations $(i i)$. From this it follows, that $u$ is $\mathcal{A}_{p}$-holomorphic in $\mathcal{A}_{p}^{n} \backslash\left(\operatorname{supp}\left(f_{1}\right) \cup \ldots \cup \operatorname{supp}\left(f_{n}\right)\right)$. In view of formula $(i i i)$ it follows, that there exists $0<r<\infty$ such that

(iv) $u(z)=0$ for each $z \in \mathcal{A}_{p}^{n}$ with $\left|z_{2}\right|+\ldots+\left|z_{n}\right|>r$. From $\partial u / \partial \tilde{z}_{1}=\hat{f}_{1}$ it follows, that $\partial u / \partial \tilde{z}_{1}=0$ in $\mathcal{A}_{p}^{n} \backslash \operatorname{supp}\left(f_{1}\right)$. Consequently, there exists $0<R<\infty$ such that $u$ may differ from 0 on $\mathcal{A}_{p}^{n} \backslash B\left(\mathcal{A}_{p}^{n}, 0, R\right)$ only on an $\mathcal{A}_{p}$ constant (see Theorem 3.28 and Note 3.11 in [20]). Together with (iv) this gives, that $u(z)=0$ on $\mathcal{A}_{p}^{n} \backslash B\left(\mathcal{A}_{p}^{n}, 0, \max (R, r)\right)$.

2.12. Theorem. Let $U$ be an open subset in $\mathcal{A}_{p}^{n}$, where $n \geq 2,2 \leq p<\infty$ or $p=\Lambda$. Suppose $K$ is a bounded closed subset in $U$ such that $U \backslash K$ is connected. Then for every $\mathcal{A}_{p}$-holomorphic function $h$ on $U \backslash K$ there exists a function $H \mathcal{A}_{p}$-holomorphic in $U$ such that $H=h$ in $U \backslash K$.

Proof. Take any infinite $(z, \tilde{z})$-differentiable function $\chi$ on $U$ with bounded closed support such that $\left.\chi\right|_{V}=1$ on some (open) neighbourhood $V$ of $K$. Then consider a family of functions $f_{j}$ such that $\hat{f}_{j}(z, \tilde{z}) . S=-\{(\partial \chi / \partial \tilde{z}) . S\} h$ in $U \backslash K$ and $f_{j}=0$ outside $U \backslash K$ for each $S$ in the set of generators of $\mathcal{A}_{p}$, where $j=1, \ldots, n, f_{j}(z)=\hat{f}_{j}(z)$.1. Therefore, conditions of Theorem 2.11 are satisfied and it gives a function $u \in C_{0,(z, \tilde{z})}^{1}\left(\mathcal{A}_{p}^{n}, \mathcal{A}_{p}\right)$ such that $\partial u / \partial \tilde{z}_{j}=\hat{f}_{j}$ for each $j=1, \ldots, n$. A desired function $H$ can be defined by the formula $H:=(1-\chi) h-u$ such that $H$ is $\mathcal{A}_{p}$-holomorphic in $U$. Since $\chi$ has a bounded closed support, then there exists an unbounded connected subset $W$ in $\mathcal{A}_{p}^{n} \backslash \operatorname{supp}(\chi)$. Therefore, 
$\left.u\right|_{W}=0$, consequently, $\left.H\right|_{U \cap W}=\left.h\right|_{U \cap W}$. From $(U \backslash K) \cap W \neq \emptyset$ and connectedness of $U \backslash K$ it follows, that $\left.H\right|_{U \backslash K}=\left.h\right|_{U \backslash K}$.

2.13. Remark. In the particular case of a singleton $K=\{z\}$ Theorem 2.12 gives nonexistence of isolated singularities, that is, each $\mathcal{A}_{p}$-holomorphic function in $U \backslash\{z\}$ for $U$ open in $\mathcal{A}_{p}^{n}$ with $n \geq 2$ can be $\mathcal{A}_{p}$-holomorphically extended to $z$. Theorem 2.12 is the $\mathcal{A}_{p}$-analog of the Hartog's theorem for $\mathbf{C}^{\mathbf{n}}$.

2.14. Corollary. Let $U$ be an open connected subset in $\mathcal{A}_{p}^{n}, 2 \leq p<\infty$ or $p=\Lambda$ and $n \geq 2$. Suppose that $f$ is a right superlinearly $\mathcal{A}_{p}$-superdifferentiable function $f: U \rightarrow \mathcal{A}_{p}$ and $N(f):=\{z \in U: f(z)=0\}$, then

(i) $U \backslash N(f)$ is connected,

(ii) $N(f)$ is not bounded closed.

Proof. Reduce the proof of this corollary to the case of finite $p$ using the beginning and the end of the proof of Theorem 2.3. Since if statements $(i, i i)$ are true in the projection from $\mathcal{A}_{\Lambda}$ on $\theta\left(\mathcal{A}_{p}\right)$, then they are true for $\mathcal{A}_{\Lambda}$. $(i)$. Write $f$ in the form $f=\sum_{l=1}^{2^{p-1}} i_{l} g_{l}$, where $g_{l}:=f_{2 l-1}+i_{2 l-1}^{*} i_{2 l} f_{2 l}, f=\sum_{s=1}^{2^{p}} i_{s-1} f_{s}, f_{s}$ are real-valued functions, $\left\{i_{0}, \ldots, i_{2^{p}-1}\right\}$ is the set of generators of the Cayley-Dickson algebra $\mathcal{A}_{p}$. In view of Proposition 2.3 and Corollary 2.5.1 [20] each function $g_{l}$ is holomorphic in complex variables $y_{k}, k=1, \ldots, 2^{p-1}$, where $z=\sum_{l=1}^{2^{p-1}} i_{2 l-1} y_{l}, y_{l}=x_{2 l-1}+i_{2 l-1}^{*} i_{2 l} x_{2 l}, x_{1}, \ldots, x_{2^{p}} \in \mathbf{R}, z \in \mathcal{A}_{p}$.

Therefore, $N(f)=\bigcap_{l=1}^{2^{p-1}} N\left(g_{l}\right)$, consequently, $U \backslash N(f)=\bigcup_{l=1}^{2^{p-1}}\left(U \backslash N\left(g_{l}\right)\right)$. Then from Corollary 1.2.4 [7] for complex holomorphic functions $(i)$ follows.

(ii). Suppose that $N(f)$ is bounded closed (compact for finite $p$ ). In view of $(i)$ and Theorem 2.12 the function $1 / f$ can be $\mathcal{A}_{p}$-holomorphically extended on $N(f)$. This is the contradiction, since $f=0$ on $N(f)$.

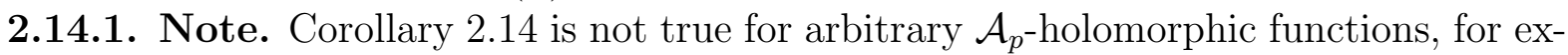
ample, $f\left({ }^{1} z,{ }^{2} z\right)=f_{1}\left({ }^{1} z\right) f_{2}\left({ }^{2} z\right)$ on $B\left(\mathcal{A}_{p}^{2}, 0,2\right)$, where $f_{1}\left({ }^{1} z\right):=-{ }^{1} z\left(\sum_{l=1}^{2^{p}} i_{l-1}{ }^{1} z i_{l-1}\right) /\left(2^{p}-\right.$ $2)-r_{1}, f_{2}\left({ }^{2} z\right):=-{ }^{2} z\left(\sum_{l=1}^{2^{p}} i_{l-1}{ }^{2} z i_{l-1}\right) /\left(2^{p}-2\right)-r_{2}$ for finite $2 \leq p, 0<r_{1}, 0<r_{2}$, $r_{1}^{2}+r_{2}^{2}<4$.

2.15. Theorem. Let $U$ be an open subset in $\mathcal{A}_{p}^{n}, 2 \leq p<\infty$ or $p=\Lambda, f_{1}, \ldots, f_{n}$ be infinite Frechét differentiable (by real variables) functions on $U$ and suppose $(z, \tilde{z})$-superdifferentiable that Conditions 2.11.( $i)$ are satisfied in $U$. Then for each open bounded polytor $P=P_{1} \times$ $\ldots \times P_{n}$ such that $c l(P)$ is a subset in $U$, there exists a function $u$ infinite differentiable (by real variables) on $P$ and satisfying Conditions 2.11.(ii) on $P$.

Proof. Using the beginning and the end of the proof of Theorem 2.3 reduce the proof of this theorem to finite $p$. Suppose that the theorem is true for $f_{m+1}=\ldots=f_{n}=0$ on $U$. The case $m=0$ is trivial. Assume that the theorem is proved for $m-1$. Consider $U^{\prime}=U^{\prime}{ }_{1} \times \ldots \times U^{\prime}{ }_{n}$ and $U^{\prime \prime}=U^{\prime \prime}{ }_{1} \times \ldots \times U^{\prime \prime}{ }_{n}$ open polytors in $\mathcal{A}_{p}^{n}$ such that $P \subset \operatorname{cl}(P) \subset$ $U^{\prime \prime} \subset \operatorname{cl}\left(U^{\prime \prime}\right) \subset U^{\prime} \subset \operatorname{cl}\left(U^{\prime}\right) \subset U$. Take an infinite differentiable (by real variables) function $\chi$ on $U^{\prime}{ }_{m}$ with compact support such that $\left.\chi\right|_{U^{\prime}{ }_{m}}=1, \chi=0$ in a neighbourhood of $\mathcal{A}_{p} \backslash U^{\prime}{ }_{m}$. There exists a function

$$
\eta(z):=-(2 \pi)^{1-2^{p}} \int_{U^{\prime} m}\left[\chi(\zeta)\left(\hat{f}_{m}\left({ }^{1} z, \ldots,{ }^{m-1} z, \zeta_{1},{ }^{m+1} z, \ldots,{ }^{n} z\right) \cdot d \tilde{\zeta}_{2^{p}}\right)\right] \wedge \nu,
$$

where a differential form $\nu$ is given in $\S 2.3$ with $\zeta_{1}, \zeta_{2}, \ldots, \zeta_{2^{p}-1} \in U^{\prime}{ }_{m}$ and ${ }^{m} z$ here for $\nu$ instead of $z$ in $\S 2.3$. By changing of variables as in $\S 2.3$ we get

$$
\eta(z):=-(2 \pi)^{1-2^{p}} \int_{\mathcal{A}_{p}}\left[\chi\left(\zeta_{1}+z\right)\left(\hat{f}_{m}\left({ }^{1} z, \ldots,{ }^{m-1} z, \zeta_{1}+{ }^{m} z,{ }^{m+1} z, \ldots,{ }^{n} z\right) . d \tilde{\zeta}_{2^{p}}\right)\right] \wedge \psi
$$

where the differential form $\psi$ is the same as in $\S 2.11$. Consequently, $\partial \eta / \partial \tilde{z}_{m}=\hat{f}_{m}$ in $U^{\prime \prime}$. In view of Conditions 2.11(i) and differentiating under the sign of the integral, since the support 
of $\chi$ is compact, we get $\partial \eta(z) / \partial \tilde{z}=\hat{f}_{j}=0$ on $U^{\prime}$ for $j=m+1, \ldots, n$, since $f_{j}=0, \hat{f}_{j} .1=f_{j}$, $\hat{f}_{j}$ is the partial (super)derivative of some function $\xi_{j}$ by $z$. Thus functions $g_{j}:=f_{j}-\partial \eta / \partial \tilde{z}$ for $j=1, \ldots, n$ fulfil the compatibility conditions $2.11(\mathrm{i})$, consequently, $g_{m}, \ldots, g_{n}=0$ in $U^{\prime \prime}$. And inevitably by the induction hypothesis there exists a function $v \in C^{\infty}\left(P, \mathcal{A}_{p}\right)$ such that $\partial v / \partial \tilde{z}_{j}=g_{j}$ in $P$ for which $u=v+\eta$ is the required solution.

2.16. Definition. Let $W$ be an open subset in $\mathcal{A}_{p}^{n}, 2 \leq p<\infty$ or $p=\Lambda$ and for each open subsets $U$ and $V$ in $\mathcal{A}_{p}^{n}$ such that

(i) $\emptyset \neq U \subset V \cap W \neq V$ and

(ii) $V$ is connected

there exists an $\mathcal{A}_{p}$-holomorphic (right superlinearly superdifferentiable, in short RSS, correspondingly) function $f$ in $W$ such that there does not exist any $\mathcal{A}_{p}$-holomorphic (RSS) function $g$ in $V$ such that $g=f$ in $U$. Then $W$ is called a domain of $\mathcal{A}_{p}$ (RSS, respectively) holomorphy. Sets of $\mathcal{A}_{p}$-holomorphic (RSS) functions in $W$ are denoted by $\mathcal{H}(W)\left(\mathcal{H}_{R S S}(W)\right.$ respectively).

2.17. Definition. Suppose that $W$ is an open subset in $\mathcal{A}_{p}^{n}, 2 \leq p<\infty$ or $p=\Lambda$ and $K$ is a closed bounded subset of $W$, then

(i) $\hat{K}_{W}^{\mathcal{H}}:=\left\{z \in W:|f(z)| \leq \sup _{\zeta \in K}\|\hat{f}(\zeta)\|\right.$ for each $\left.f \in \mathcal{H}(W)\right\}$;

(ii) $\hat{K}_{W}^{\mathcal{H}_{R S S}}:=\left\{z \in W:|f(z)| \leq \sup _{\zeta \in K}|f(\zeta)|\right.$ for each $\left.f \in \mathcal{H}_{R S S}(W)\right\}$;

these sets are called the $\mathcal{H}(W)$-convex hull of $K$ and the $\mathcal{H}_{R S S}(W)$-convex hull of $K$ respectively, where $\|\hat{f}(\zeta)\|:=\sup _{h \in \mathcal{A}_{p}^{n},|h| \leq 1}|\hat{f}(\zeta) . h|$. If $K=\hat{K}_{W}^{\mathcal{H}}$ or $K=\hat{K}_{W}^{\mathcal{H}_{R S S}}$, then $K$ is called $\mathcal{H}(W)$-convex or $\mathcal{H}_{R S S}(W)$-convex correspondingly.

2.18. Proposition. For each closed bounded subset $K$ in $\mathcal{A}_{p}^{n}, 2 \leq p<\infty$ or $p=\Lambda$, the $\mathcal{H}\left(\mathcal{A}_{p}^{n}\right)$-hull and $\mathcal{H}_{R S S}\left(\mathcal{A}_{p}^{n}\right)$-hull of $K$ are contained in the $\mathbf{R}$-convex hull of $K$.

Proof. Reduce the proof to the case of finite $p$ using $§ 2.3$.

I. Consider at first the $\mathcal{H}\left(\mathcal{A}_{p}^{n}\right)$-hull of $K$. Each $z \in \mathcal{A}_{p}^{n}$ can be written in the form $z=\left({ }^{1} z, \ldots,{ }^{n} z\right),{ }^{j} z \in \mathcal{A}_{p},{ }^{j} z=\sum_{l=1}^{2^{p}} x_{l, j} S_{l}$, where $x_{l, j}=x_{l, j}(z) \in \mathbf{R}, S_{l}=i_{l-1}$. If $w \in \mathcal{A}_{p}^{n}$, $w \notin c o_{\mathbf{R}}(K)$, then there are $y_{1}, \ldots, y_{2^{p} n} \in \mathbf{R}$ such that $\sum_{j=1}^{n} \sum_{l=1}^{2^{p}} x_{l, j}(w) y_{2^{p}(j-1)+l}=0$, but $\sum_{j=1}^{n} \sum_{l=1}^{2^{p}} x_{l, j}(w) y_{2^{p}(j-1)+l}<0$ if $z \in K$, where $c o_{\mathbf{R}}(K):=\left\{z \in \mathcal{A}_{p}^{n}\right.$ : there are $a_{1}, \ldots, a_{s} \in \mathbf{R}$ and $v_{1}, \ldots, v_{s} \in K$ such that $\left.z=a_{1} v_{1}+\ldots+a_{k} v_{k}\right\}$ denotes a R-convex hull of $K$ in $\mathcal{A}_{p}^{n}$. Put $\zeta_{j}=\sum_{l, j} y_{2^{p}(j-1)+l} S_{l}$, then $f(z):=\exp \left(\sum_{j=1}^{n} z_{j} \tilde{\zeta}_{j}\right)$ is the $\mathcal{A}_{p}$-holomorphic function in $\mathcal{A}_{p}^{n}$ such that $|f(z)|<1$ for each $z \in K$ and $|f(w)|=1$ for the marked point $w$ above (see Corollary $3.3[19]$ ), since $i_{v}^{2}=-1$ for each $v>0$. From $\|\hat{f}(\zeta)\| \geq|f(\zeta)|$ the first statement follows.

$I I$. Consider now the $\mathcal{H}_{R S S}\left(\mathcal{A}_{p}^{n}\right)$-hull of $K$. Each $f \in \mathcal{H}_{R S S}(W)$ has the form $f=$ $\sum_{l=1}^{2^{p-1}} i_{l} g_{l}$, where each function $g_{l}$ is holomorphic in complex variables $y_{k}$ (see $\S 2.14$ ).

The set $K$ has projection $K_{k}$ on the complex subspaces $\mathbf{C}^{\mathbf{n}}$ corresponding to variables

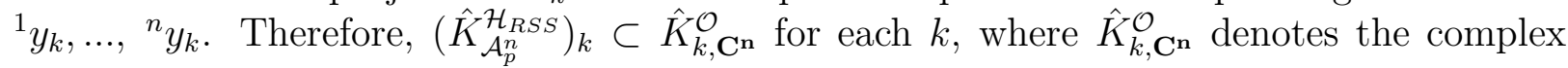
holomorphic hull of $K_{k}$ in $\mathbf{C}^{\mathbf{n}}$. In view of Proposition 1.3.3 [7] $\hat{K}_{k, \mathbf{C}^{\mathbf{n}}}^{\mathcal{O}} \subset \operatorname{co}_{\mathbf{R}}\left(K_{k}\right)$, hence $\hat{K}_{\mathcal{A}_{p}^{n}}^{\mathcal{H}_{R S S}} \subset \mathrm{co}_{\mathbf{R}}(K)$.

2.18.1. Note. Due to Proposition 2.18 above Corollary 1.3.4 [7] can be transferred on $\mathcal{H}$ and $\mathcal{H}_{R S S}$ for $\mathcal{A}_{p}^{n}$ instead of $\mathbf{C}^{\mathbf{n}}$. Also $\mathcal{A}_{p}$-versions of Theorems 1.3.5, 7, 11, Corollaries 1.3.6, 8, 9, 10,13 and Definition 1.3 .12 are true in the $\mathcal{H}_{R S S^{-}}$class of functions instead of complex holomorphic functions. 


\section{Integral representations of functions of Cayley-Dickson variables}

3.1. Definitions and Notations. Consider an $\mathcal{A}_{p^{-}}$valued function on $\mathcal{A}_{p}^{n}, 2 \leq p<\infty$ or $p=\Lambda$ such that

(i) $(\zeta, \zeta)=a e$ with $a \geq 0$ and $(\zeta, \zeta)=0$ if and only if $\zeta=0$,

(ii) $(\zeta, z+\xi)=(\zeta, z)+(\zeta, \xi)$,

(iii) $(\zeta+\xi, z)=(\zeta, z)+(\xi, z)$,

(iv) $(\alpha \zeta, z)=\alpha(\zeta, z)=(\zeta, \alpha z)$ for each $\alpha \in \mathbf{R}$ and $(\zeta \alpha, \zeta)=\tilde{\alpha}(\zeta, \zeta)$ for each $\alpha \in \mathcal{A}_{p}$,

(v) $(\zeta, z)^{\tilde{*}}=(z, \zeta)$ for each $\zeta, \xi$ and $z \in \mathcal{A}_{p}^{n}, n \in \mathbf{N}$. Then this function is called the scalar product in $\mathcal{A}_{p}^{n}$. The corresponding norm is:

(vi) $|\zeta|=\{(\zeta, \zeta)\}^{1 / 2}$. In particular, it is possible to take the canonical scalar product:

(vii) $\quad\left\langle\zeta ; z>:=(\zeta, z)=\sum_{l=1}^{n}{ }^{l} \tilde{\zeta}^{l} z\right.$, where $z=\left({ }^{1} z, \ldots,{ }^{n} z\right),{ }^{l} z \in \mathcal{A}_{p}$.

Consider differential forms on $\mathcal{A}_{p}$ :

(1) $\phi_{p, 0}(z):=d \tilde{z} \wedge d \tilde{z}, \phi_{p, 0}^{\prime}(z):=\tilde{z} d \tilde{z}$

$\phi_{p, k}(z):=\left(i_{2 k}\left(d \tilde{z} i_{2 k}\right)\right) \wedge\left(i_{2 k}\left(d z i_{2 k}\right)\right), \phi_{p, k}^{\prime}(z):=\left(i_{2 k}\left(\tilde{z} i_{2 k}\right)\right) \wedge\left(i_{2 k}\left(d z i_{2 k}\right)\right)$, for each $k=$ $1, \ldots, 2^{p-1}-1$,

(2) $w_{2^{p}}(z):=C_{p}\left\{\phi_{p, 0}(z) \wedge \phi_{p, 1}(z) \wedge \ldots \wedge \phi_{p, 2^{p-1}-1}(z)\right\}_{q_{0}\left(2^{p-1}\right)}$,

where $C_{p}=$ const $\neq 0$;

(3) $w_{2^{p}, k}(\zeta-z):=\left\{\phi_{p, 0}(\zeta) \wedge \ldots \wedge \phi_{p, k-1}(\zeta) \wedge \phi_{p, k}^{\prime}(\zeta-z) \wedge \phi_{p, k+1}(\zeta) \wedge \phi_{p, 2^{p-1}-1}(\zeta)\right\}_{q_{0}\left(2^{p-1}\right)}$ for each $k=0, \ldots, 2^{p-1}-1$,

where $q_{0}(s)$ means the associated product in definite order corresponding to the left preferred order of brackets, $\left\{b_{1} \ldots b_{s}\right\}_{q_{0}(s)}:=\left(\ldots\left(\left(b_{1} b_{2}\right) b_{3}\right) \ldots b_{s-1}\right) b_{s}$ for $b_{1}, \ldots, b_{s} \in \mathcal{A}_{p}$. Introduce also differential forms:

(4) $\check{\phi}_{p, 0}(\zeta, z):=(d \tilde{\zeta}-d \tilde{z}) \wedge d \tilde{\zeta}, \check{\phi}_{p, 0}^{\prime}(\zeta, z):=(\tilde{\zeta}-\tilde{z}) d \tilde{\zeta}$

$\left.\check{\phi}_{p, k}(\zeta, z):=\left(i_{2 k}(d \tilde{\zeta}-d \tilde{z}) i_{2 k}\right)\right) \wedge\left(i_{2 k}\left(d \zeta i_{2 k}\right)\right), \check{\phi}_{p, k}^{\prime}(\zeta, z):=\left(i_{2 k}\left((\tilde{\zeta}-\tilde{z}) i_{2 k}\right)\right) \wedge\left(i_{2 k}\left(d \zeta i_{2 k}\right)\right)$, for each $k=1, \ldots, 2^{p-1}-1$,

(5) $\check{w}_{2^{p}}(\zeta, z):=C_{p}\left\{\check{\phi}_{p, 0}(\zeta, z) \wedge \check{\phi}_{p, 1}(\zeta, z) \wedge \ldots \wedge \check{\phi}_{p, 2^{p-1}-1}(\zeta, z)\right\}_{q_{0}\left(2^{p-1}\right)}$, where $C_{p}=\left(\left[\left(2^{p}-2\right) !\right] 2\left(2^{p-1}-1\right)\right)^{-1}$;

(6) $\quad \check{w}_{2^{p}, k}(\zeta, z):=\left\{\check{\phi}_{p, 0}(\zeta, z) \wedge \ldots \wedge \check{\phi}_{p, k-1}(\zeta, z) \wedge \check{\phi}_{p, k}^{\prime}(\zeta, z) \wedge \check{\phi}_{p, k+1}(\zeta, z) \wedge \ldots \wedge \check{\phi}_{p, 2^{p-1}-1}(\zeta, z)\right\}_{q_{0}\left(2^{p-1}\right)}$ for each $k=0, \ldots, 2^{p-1}-1$,

(7) $\hat{\phi}_{p, 0}(\zeta, z):=(d \tilde{\zeta}-d \tilde{z}) \wedge(d \tilde{\zeta}-d \tilde{z}), \hat{\phi}_{p, 0}^{\prime}(\zeta, z):=(\tilde{\zeta}-\tilde{z})(d \tilde{\zeta}-d \tilde{z})$,

$\left.\left.\hat{\phi}_{p, k}(\zeta, z):=\left(i_{2 k}(d \tilde{\zeta}-d \tilde{z}) i_{2 k}\right)\right) \wedge\left(i_{2 k}\left((d \zeta-d z) i_{2 k}\right)\right)\right), \hat{\phi}_{p, k}^{\prime}(\zeta, z):=\left(i_{2 k}\left((\tilde{\zeta}-\tilde{z}) i_{2 k}\right)\right) \wedge\left(i_{2 k}((d \zeta-\right.$ $\left.d z) i_{2 k}\right)$ ), for each $k=1, \ldots, 2^{p-1}-1$,

(8) $\hat{w}_{2^{p}}(\zeta, z):=C_{p}\left\{\hat{\phi}_{p, 0}(\zeta, z) \wedge \hat{\phi}_{p, 1}(\zeta, z) \wedge \ldots \wedge \hat{\phi}_{p, 2^{p-1}-1}(\zeta, z)\right\}_{q_{0}\left(2^{p-1}\right)}$, where $C_{p}=\left(\left[\left(2^{p}-2\right) !\right] 2\left(2^{p-1}-1\right)\right)^{-1}$;

(9) $\hat{w}_{2^{p}, k}(\zeta, z):=\left\{\hat{\phi}_{p, 0}(\zeta, z) \wedge \ldots \wedge \hat{\phi}_{p, k-1}(\zeta, z) \wedge \hat{\phi}_{p, k}^{\prime}(\zeta, z) \wedge \hat{\phi}_{p, k+1}(\zeta, z) \wedge \ldots \wedge \hat{\phi}_{p, 2^{p-1}-1}(\zeta, z)\right\}_{q_{0}\left(2^{p-1}\right)}$ for each $k=0, \ldots, 2^{p-1}-1$,

where we can express $\tilde{\zeta}$ and $\tilde{z}$ in the $\zeta$ and $z$-representations respectively: $\tilde{z}=\left(2^{p}-2\right)^{-1}\{-z+$ $\left.\sum_{s \in \hat{b}} s(z \tilde{s})\right\}$ for each $2 \leq p \in \mathbf{N}$. With the help of them construct differential forms on $\mathcal{A}_{p}^{n}$ :

$$
\begin{gathered}
\text { (10) } \theta_{z}(\zeta):=C^{\prime}{ }_{p}|\zeta-z|^{-2^{p} n} \sum_{s=1}^{n} \sum_{q=0}^{2^{p-1}-1}\left\{w_{2^{p}}\left({ }^{1} \zeta\right) \wedge \ldots\right. \\
\left.\wedge w_{2^{p}}\left({ }^{s-1} \zeta\right) \wedge w_{2^{p}, q}\left({ }^{s} \zeta-{ }^{s} z\right) \wedge w_{2^{p}}\left({ }^{s+1} \zeta\right) \wedge \ldots \wedge w_{2^{p}}\left({ }^{n} \zeta\right)\right\}_{q_{0}(n)} \\
(11) \quad \check{\theta}(\zeta, z):=C^{\prime}{ }_{p}|\zeta-z|^{-2^{p} n} \sum_{s=1}^{n} \sum_{q=0}^{2^{p-1}-1}\left\{\check{w}_{2^{p}}\left({ }^{1} \zeta,{ }^{1} z\right) \wedge \ldots\right.
\end{gathered}
$$




$$
\begin{gathered}
\left.\wedge \check{w}_{2^{p}}\left({ }^{s-1} \zeta,{ }^{s-1} z\right) \wedge \check{w}_{2^{p}, q}\left({ }^{s} \zeta,{ }^{s} z\right) \wedge \check{w}_{2^{p}}\left({ }^{s+1} \zeta,{ }^{s+1} z\right) \wedge \ldots \wedge \check{w}_{2^{p}}\left({ }^{n} \zeta,{ }^{n} z\right)\right\}_{q_{0}(n)} \\
(12) \quad \hat{\theta}(\zeta, z):=C^{\prime}{ }_{p}|\zeta-z|^{-2^{p} n} \sum_{s=1}^{n} \sum_{q=0}^{2^{p-1}-1}\left\{\hat{w}_{2^{p}}\left({ }^{1} \zeta,{ }^{1} z\right) \wedge \ldots\right. \\
\left.\wedge \hat{w}_{2^{p}}\left({ }^{s-1} \zeta,{ }^{s-1} z\right) \wedge \hat{w}_{2^{p}, q}\left({ }^{s} \zeta,{ }^{s} z\right) \wedge \hat{w}_{2^{p}}\left({ }^{s+1} \zeta,{ }^{s+1} z\right) \wedge \ldots \wedge \hat{w}_{2^{p}}\left({ }^{n} \zeta,{ }^{n} z\right)\right\}_{q_{0}(n)},
\end{gathered}
$$

where $C_{p}^{\prime}:=\left(2^{p} n\right) ! !(2 \pi)^{-2^{p-1} n} ;|\zeta-z|^{2}$ is considered in the $(\zeta-z, \tilde{\zeta}-\tilde{z})$-representation: $\left|{ }^{s} \zeta-{ }^{s} z\right|^{2}=\left({ }^{s} \zeta-{ }^{s} z\right)\left({ }^{s} \tilde{\zeta}-{ }^{s} \tilde{z}\right),|\zeta-z|^{2}=\sum_{s=1}^{n}\left|{ }^{s} \zeta-{ }^{s} z\right|^{2}, \zeta$ and $z \in \mathcal{A}_{p}^{n}$. If $U$ is an open subset in $\mathcal{A}_{p}^{n}$ and $f$ is a bounded $\mathcal{A}_{p}$-differential form on $U$, then by the definition:

$$
(13) \quad\left(\mathcal{B}_{U} f\right)(z):=\int_{\zeta \in U} f(\zeta) \wedge \check{\theta}(\zeta, z)
$$

for each $z \in \mathcal{A}_{p}^{n}$. If in addition $U$ is with a continuous piecewise $C^{1}$-boundary (by the corresponding real variables) and $f$ is a bounded differential form on $\partial U$, then by the definition:

$$
(14) \quad\left(\mathcal{B}_{\partial U} f\right)(z):=\int_{\zeta \in \partial U} f(\zeta) \wedge \check{\theta}(\zeta, z)
$$

for each $z \in \mathcal{A}_{p}^{n}$.

3.2. Theorem. Let $U$ be an open subset in $\mathcal{A}_{p}^{n}, 2 \leq p \in \mathbf{N}$, with piecewise $C^{1}$-boundary $\partial U$. Suppose that $f$ is a continuous function on $\operatorname{cl}(U)$ and $\tilde{\partial} f$ is continuous on $U$ in the sense of distributions and has a continuous extension on $\operatorname{cl}(U)$. Then

$$
\text { (1) } f=\mathcal{B}_{\partial U} f-\mathcal{B}_{U} \tilde{\partial} f \text { on } U
$$

where $\mathcal{B}_{U}$ and $\mathcal{B}_{\partial U}$ are the $\mathcal{A}_{p}$-integral operators given by Equations 3.1.(13, 14).

Proof. Write the variable $z$ in the form $z=\sum_{l=0}^{2^{p-1}-1} i_{2 l} \alpha_{l}$, where $\alpha_{l} \in \mathbf{C}_{l}:=\mathbf{R} \oplus i_{2 l}^{*} i_{2 l+1} \mathbf{R}$, $i_{2 l} \alpha_{l}=i_{2 l} x_{l}+i_{2 l+1} y_{l}$, where $x_{l}, y_{l} \in \mathbf{R}$. Then

(1) $\alpha_{0} i_{k}=i_{k} \bar{\alpha}_{0}$ for each $k>0$,

(2) $i_{2 k}\left(d z i_{2 k}\right)=\left(\sum_{l>0, l \neq k} i_{2 l} d \alpha_{l}\right)-i_{2 k} d \bar{\alpha}_{k}-d \bar{\alpha}_{0}$,

(3) $\quad\left(i_{2 l} d \alpha_{l}\right) \wedge d \bar{\alpha}_{0}=-d \alpha_{0} \wedge\left(i_{2 l} d \alpha_{l}\right)$ for each $l>0$,

(4) $\left(i_{2 l} d \alpha_{l}\right) \wedge\left(i_{2 q} d \alpha_{q}\right)=\left(i_{2 q} d \alpha_{q}\right) \wedge\left(i_{2 l} d \alpha_{l}\right)$ for each $l \neq q$ with $l>0$ and $q>0$,

(5) $\left(i_{2 l} d \alpha_{l}\right) \wedge\left(i_{2 l} d \bar{\alpha}_{l}\right)=0$ for each $l>0$, then

(6) $d \alpha_{0} \wedge d \bar{\alpha}_{0}=-d \bar{\alpha}_{0} \wedge d \alpha_{0}=-2 i_{1} d x_{0} \wedge d y_{0}, d \alpha_{0} \wedge d \alpha_{0}=0$

(7) $\left(i_{2 l} d \alpha_{l}\right) \wedge\left(i_{2 l} d \alpha_{l}\right)=2 i_{2 l} i_{2 l+1} d x_{l} \wedge d y_{l}, d \alpha_{l} \wedge d \bar{\alpha}_{l}=-2 i_{2 l}^{*} i_{2 l+1} d x_{l} \wedge d y_{l}=2 i_{2 l} i_{2 l+1} d x_{l} \wedge d y_{l}$ for each $l>0$.

From Equations $(1-7)$ and $d \tilde{z}=d \bar{\alpha}_{0}-\sum_{l>0} i_{2 l} d \alpha_{l}$ it follows, that

(8) $\phi_{p, 0}(z)=\left(d \alpha_{0}-d \bar{\alpha}_{0}\right) \wedge\left(\sum_{q>0} i_{2 q} d \alpha_{q}\right)+\sum_{l>0} \sum_{q>0}\left(i_{2 l} d \alpha_{l}\right) \wedge\left(i_{2 q} d \alpha_{q}\right)$,

(9) $\phi_{p, k}(z)=\left(d \alpha_{0} \wedge d \bar{\alpha}_{0}\right)-\left(d \alpha_{k} \wedge d \bar{\alpha}_{k}\right)+\left(\left(2 i_{2 k} d \bar{\alpha}_{k}\right) \wedge\left(\sum_{l>0, l \neq k} i_{2 l} d \alpha_{l}\right)\right)-\sum_{l>0, l \neq k} \sum_{q>0, q \neq k}\left(i_{2 l} d \alpha_{l}\right) \wedge$ $\left(i_{2 q} d \alpha_{q}\right)$

for each $k>0$. The differential form $w_{2^{p}}$ is of degree $2^{p}$ in real coordinates $x_{0}, y_{0}, \ldots$, $x_{2^{p-1}-1}, y_{2^{p-1}-1}$, hence it may contain only the multiplier $d x_{0} \wedge d y_{0}$ or may contain only $d \alpha_{0} \wedge d \bar{\alpha}_{0}$, hence all terms in $w_{2^{p}}$ arising from the term $\left(d \alpha_{0}-d \bar{\alpha}_{0}\right) \wedge\left(\sum_{q>0} i_{2 q} d \alpha_{q}\right)$ in $\phi_{p, 0}(z)$ cancel, since $\phi_{p, k}(z)$ contains $d \alpha_{0} \wedge d \bar{\alpha}_{0}$ for each $k>0$. Then from $(3-5)$ it follows, that all terms arising from the term $\left(\left(2 i_{2 k} d \bar{\alpha}_{k}\right) \wedge\left(\sum_{l>0, l \neq k} i_{2 l} d \alpha_{l}\right)\right)$ in $\phi_{p, k}(z)$ for $k>0$ cancel in $w_{2^{p}}$. Thus for a choice of the multiplier $d \alpha_{0} \wedge d \bar{\alpha}_{0}$ in $w_{2^{p}}$ there are $\left(2^{p-1}-1\right)$ possibilities among $\phi_{p, q}(z)$ with $q=1, . ., 2^{p-1}-1$ in the graded external product. After a choice of $d \alpha_{0} \wedge d \bar{\alpha}_{0}$ for some $q>0$ it remains $\left(2^{p}-2\right)\left(2^{p}-3\right) / 2$ variants for a choice of the multiplier $d \alpha_{1} \wedge d \bar{\alpha}_{1}=\left(i_{2} d \alpha_{1}\right) \wedge\left(i_{2} d \alpha_{1}\right)$. Then by induction after choices of the multipliers $\left(i_{2 v} d \alpha_{v}\right) \wedge\left(i_{2 v} d \alpha_{v}\right)=d \alpha_{v} \wedge d \bar{\alpha}_{v}$ for $v=0,1, \ldots, q-1$ with $q>2$ it remains 
$\left(2^{p}-2 q\right)\left(2^{p}-2 q-1\right) / 2$ variants for choices of the multiplier $\left(i_{2 q} d \alpha_{q}\right) \wedge\left(i_{2 q} d \alpha_{q}\right)$. Thus

(10) $w_{2^{p}}=(-1)^{2^{p-1}-2} C_{p}\left[\left(2^{p}-2\right) !\right]\left(2^{p-1}-1\right) 2^{-\left(2^{p-1}-1\right)}\left\{\left(d \alpha_{0} \wedge d \bar{\alpha}_{0}\right) \wedge\left(\left(i_{2} d \alpha_{1}\right) \wedge\left(i_{2} d \alpha_{1}\right)\right) \wedge\right.$ $\left.\cdots \wedge\left(\left(i_{2^{p}-2} d \alpha_{2^{p-1}-1}\right) \wedge\left(i_{2^{p}-2} d \alpha_{2^{p-1}-1}\right)\right)\right\}_{q_{0}\left(2^{p-1}\right)}$

$=\left(C_{p}\left[\left(2^{p}-2\right) !\right] 2\left(2^{p-1}-1\right)\right) d x_{0} \wedge d y_{0} \wedge d x_{1} \wedge d y_{1} \wedge \ldots \wedge d x_{2^{p-1}-1} \wedge d y_{2^{p-1}-1}$,

since $\left(i_{0} i_{1}\right)\left(i_{2} i_{3}\right)=i_{1}^{2}=-1,\left\{\left(i_{0} i_{1}\right)\left(i_{2} i_{3}\right) \ldots\left(i_{2^{p-2}} i_{2^{p-1}}\right)\right\}_{q_{0}\left(2^{p-1}\right)}=-1$. Hence $w_{2^{p}}$ is the volume element on $\mathcal{A}_{p}$ equal to the Lebesgue measure $\mu$ on the underlying Euclidean space $\mathbf{R}^{2^{p}}$ such that $\mu\left([0,1]^{2^{p}}\right)=1$, since $C_{p}=\left(\left[\left(2^{p}-2\right) !\right] 2\left(2^{p-1}-1\right)\right)^{-1}$.

The differential form $\check{\theta}(\zeta, z)$ has the decomposition

$$
\check{\theta}(\zeta, z)=\sum_{q=0}^{2^{p-1} n-1} \Upsilon_{q}(\zeta, z)
$$

where $\Upsilon_{q}(\zeta, z)$ is the $\mathcal{A}_{p}$-differential form with all terms of degree $2^{p} n-q-1$ by $\zeta$ and $\tilde{\zeta}$ and their multiples on $\mathcal{A}_{p}$ constants and of degree $q$ by $z$ and $\tilde{z}$ and their multiples on $\mathcal{A}_{p}$ constants. The differential form $f(\zeta)$ has the decomposition

$$
f(\zeta)=\sum_{r=0}^{m} f_{r}(\zeta)
$$

where $m=\operatorname{deg}(f), f_{r}(\zeta)$ is with all terms of degree $r$ by $\zeta$ and $\tilde{\zeta}$ and their multiples on $\mathcal{A}_{p}$ constants. Then $f_{r} \wedge \Upsilon_{q}=0$, when $r>q+1$. By the definition of integration $\int_{\zeta \in U} f_{r}(\zeta) \wedge \Upsilon_{q}(\zeta, z)=0$ for $r<q+1$. If $f$ is a function, then $\int_{\zeta \in \partial U} f(\zeta) \Upsilon_{q}(\zeta, z)=0$ for each $q>0$, since $\partial U$ has the dimension $2^{p} n-1$, hence

$$
\left(\mathcal{B}_{\partial U} f\right)(z)=\int_{\zeta \in \partial U} f(\zeta) \theta_{z}(\zeta)
$$

since $\Upsilon_{0}(\zeta, z)=\theta_{z}(\zeta)$. If $f$ is a 1 -form, then $\int_{\zeta \in U} f(\zeta) \wedge \Upsilon_{q}(\zeta, z)=0$ for each $q>0$, since $U$ has the dimension $2^{p} n$, consequently,

$$
\left(\mathcal{B}_{U} f\right)(z)=\int_{\zeta \in U} f(\zeta) \wedge \theta_{z}(\zeta)
$$

In particular, there are identities in $\mathbf{H}: d \xi \wedge j d \zeta=d \xi j \wedge d \zeta$ and $(\xi d \zeta)^{\tilde{*}}=[d \tilde{\zeta}] \tilde{\xi}$ for each $\xi, \zeta \in \mathbf{H}$. Then

(i) $d \zeta \wedge j d \tilde{\zeta} \wedge d \tilde{\zeta} \wedge d \zeta=0$

(ii) $d \zeta \wedge d \tilde{\zeta} \wedge j d \tilde{\zeta} \wedge d \zeta=0$

(iii) $\quad d \zeta \wedge d \tilde{\zeta} \wedge d \tilde{\zeta} \wedge j d \zeta=0$, since $j^{2}=-e$ and $\mathbf{R} e$ is the centre of the quaternion algebra $\mathbf{H}, \alpha$ and $\beta \in \mathbf{C}$ commute with $d \alpha, d \bar{\alpha}, d \beta$ and $d \bar{\beta}$, where $\zeta=\alpha e+\beta j$. From $(i-i i i)$ with the help of bijective surjective mappings $\zeta \mapsto j \zeta$ and $\zeta \mapsto \zeta j$ it follows, that

(iv) $\quad d \zeta \wedge d \tilde{\zeta} \wedge d \tilde{\zeta} \wedge d \zeta=0$

(v) $d \zeta \wedge j d \tilde{\zeta} \wedge j d \tilde{\zeta} \wedge d \zeta=0$

(vi) $\quad d \zeta \wedge j d \tilde{\zeta} \wedge j d \tilde{\zeta} j \wedge j d \zeta j=0$.

Write $\xi \in \mathcal{A}_{p}$ in the form $\xi=\alpha+\beta l$, then $\tilde{\xi}=\tilde{\alpha}-\beta l$, where $\alpha \in \mathcal{A}_{p-1}$ and $\beta \in \mathcal{A}_{p-1}$, $l$ is the generator of the doubling procedure of $\mathcal{A}_{p}$ from $\mathcal{A}_{p-1}[1]$, since there is the identity $\beta l=l \tilde{\beta}$. The decomposition $\xi=\alpha+\beta l$ is unique for each $\xi \in \mathcal{A}_{p}$, where $\alpha=\alpha(\xi)$ and $\beta=\beta(\xi)$ depend on $\xi$ in general. Put

$\kappa_{p, 0}(z):=d z \wedge d \tilde{z}$ and

$\kappa_{p, q}(z):=\left(i_{2 q}\left(d z i_{2 q}\right)\right) \wedge\left(i_{2 q}\left(d z i_{2 q}\right)\right)$ for each $1 \leq q \leq 2^{p-1}-1$. From Formulas $(1-9)$ or from Formulas $(i-v i)$ and induction by $p$ with the help of doubling procedures it follows, that 
(15)

$$
\left\{\phi_{p, 0}(z) \wedge \ldots \wedge \phi_{p, v-1}(z) \wedge \kappa_{p, v}(z) \wedge \phi_{p, v+1}(z) \wedge \ldots \wedge \phi_{p, 2^{p-1}-1}(z)\right\}_{q_{0}\left(2^{p-1}\right)}=0
$$

for each $v=0,1, \ldots, 2^{p-1}-1$.

In the $(\zeta-z, \tilde{\zeta}-\tilde{z})$-representation

$|\zeta-z|^{2}=\sum_{s=1}^{n}\left({ }^{s} \tilde{\zeta}-{ }^{s} \tilde{z}\right)\left({ }^{s} \zeta-{ }^{s} z\right)$, hence:

(16) $d_{\zeta}|\zeta-z|^{p^{p} n}=\left(2^{p-1} n\right)|\zeta-z|^{2^{p} n-2} \sum_{s=1}^{n}\left\{\left(d^{s} \tilde{\zeta}\right)\left({ }^{s} \zeta-{ }^{s} z\right)+\left({ }^{s} \tilde{\zeta}-{ }^{s} \tilde{z}\right) d^{s} \zeta\right\}$.

From Formulas $(15,16)$ it follows, that

$$
d_{\zeta}\left(|\zeta-z|^{2^{p}} \theta_{z}(\zeta)\right)=C^{\prime}{ }_{p} 2^{p-1} n\left\{w_{2^{p}}\left({ }^{1} \zeta\right) \wedge \ldots \wedge w_{2^{p}}\left({ }^{n} \zeta\right)\right\}_{q_{0}(n)}
$$

since $d_{\zeta}=\partial_{\zeta}+\partial_{\tilde{\zeta}}$. Now calculate $d_{\zeta} \theta_{z}(\zeta)$ in $U \backslash\{z\}$ using Formulas $(15-17)$ :

(18) $d_{\zeta} \theta_{z}(\zeta)=0$.

There exists $\epsilon_{0}>0$ such that for each $0<\epsilon<\epsilon_{0}$ the ball $B\left(\mathcal{A}_{p}^{n}, z, \epsilon\right):=\left\{\zeta \in \mathcal{A}_{p}^{n}\right.$ : $|\zeta-z| \leq \epsilon\}$ and hence the sphere $S\left(\mathcal{A}_{p}^{n}, z, \epsilon\right):=\left\{\zeta \in \mathcal{A}_{p}^{n}:|\zeta-z|=\epsilon\right\}=\partial B\left(\mathcal{A}_{p}^{n}, z, \epsilon\right)$ are contained in $U$. Apply the Stoke's formula for vector-valued functions and differential forms componentwise, using the Euclidean space $\mathbf{R}^{\mathbf{2}^{\mathbf{P}} \mathbf{n}}$ underlying $\mathcal{A}_{p}^{n}$, then

(19) $\int_{S\left(\mathcal{A}_{p}^{n}, z, \epsilon\right)} f(\zeta) \theta_{z}(\zeta)=\int_{\partial U} f(\zeta) \theta_{z}(\zeta)-\int_{U_{\epsilon}}[d f(\zeta)] \wedge \theta_{z}(\zeta)$, where $U_{\epsilon}:=U \backslash B\left(\mathcal{A}_{p}^{n}, z, \epsilon\right)$, $0<\epsilon<\epsilon_{0}$. Therefore, from $(15,18)$ it follows, that

(20) $\mathcal{B}_{U} d f=\mathcal{B}_{U} \tilde{\partial} f$, since $d f=\partial f+\tilde{\partial} f$, where $\partial f(\zeta)=(\partial f(\zeta) / \partial \zeta) . d \zeta, \tilde{\partial} f(\zeta)=$ $(\partial f(\zeta) / \partial \tilde{\zeta}) . d \tilde{\zeta}, f(\zeta)=f(\zeta, \tilde{\zeta})$ is the abbreviated notation.

In view of Formula (18) and the Stoke's formula:

$$
\int_{S\left(\mathcal{A}_{p}^{n}, z, \epsilon\right)} \theta_{z}(\zeta)=\left[(2 \pi)^{2^{p-1} n} /\left(2^{p} n\right) ! !\right]^{-1}\left[\epsilon^{-4 n}\right] \int_{B\left(\mathcal{A}_{p}^{n}, z, \epsilon\right)}(d V) e=e, \text { where } d V \text { is the stan- }
$$

dard volume element of the Euclidean space $\mathbf{R}^{\mathbf{2}^{\mathbf{p}} \mathbf{n}}$. In the even dimensional Euclidean space $\mathbf{R}^{2 k}$ the volume $V_{2 k}$ of the ball of radius 1 relative to the standard Lebesgue measure $\lambda$ with $\lambda\left([0,1]^{2 k}\right)=1$ is $V_{2 k}=(2 \pi)^{k} /(2 k) ! !$ (see $\S X I .4 .2$, Example 3, in [27]). Then Formula (21) implies, that

$\lim _{\epsilon \rightarrow 0} \int_{S\left(\mathcal{A}_{p}^{n}, z, \epsilon\right)} f(\zeta) \theta_{z}(\zeta)=f(z)$, since

$\int_{S\left(\mathcal{A}_{p}^{n}, z, \epsilon\right)}(f(\zeta)-f(z)) \theta_{z}(\zeta)=\epsilon^{-2^{p} n+1} \int_{S\left(\mathcal{A}_{p}^{n}, z, \epsilon\right)}(f(\zeta)-f(z))\left[|\zeta-z|^{2^{p} n-1} \theta_{z}(\zeta)\right]$. The form $[\mid \zeta-$ $\left.\left.z\right|^{2^{p} n-1} \theta_{z}(\zeta)\right]$ is bounded on $U$, consequently, $\left|\int_{S\left(\mathcal{A}_{p}^{n}, z, \epsilon\right)}(f(\zeta)-f(z)) \theta_{z}(\zeta)\right| \leq C_{1} \max \{\mid f(\zeta)-$ $\left.f(z) \mid: \zeta \in B\left(\mathcal{A}_{p}^{n}, z, \epsilon\right)\right\}$, where $C_{1}$ is a positive constant independent of $f$ and $\epsilon$ for each $0<\epsilon<\epsilon_{0}$. Therefore, Formula (1) follows from Formula (19) by taking the limit when $\epsilon>0$ tends to zero and using Identity (20).

3.3. Corollary. Let $U$ be an open subset in $\mathcal{A}_{p}^{n}, 2 \leq p \in \mathbf{N}$, and $f$ be a continuous function on $\mathrm{cl}(U)$ and $\mathcal{A}_{p}$-holomorphic on $U$. Then

(1) $f=\mathcal{B}_{\partial U} f$ on $U$,

where $\mathcal{B}_{U}$ and $\mathcal{B}_{\partial U}$ are the integral operators given by Equations 3.1. $(13,14)$.

Proof. From $\tilde{\partial} f=0$, since $\partial f(\zeta) / \partial \tilde{\zeta}=0$, and Formula 3.2.(1) implies Formula 3.3.(1).

3.4. Definitions and Notations. Suppose that $U$ is a bounded open subset in $\mathcal{A}_{p}^{n}$ and $\psi(\zeta, z)$ be an $\mathcal{A}_{p}$-valued $C^{1}$-function (by the corresponding real variables) defined on $V \times U$, where $V$ is a neighbourhood of $\partial U$ in $\mathcal{A}_{p}^{n}$, such that

(1) $\left\langle\psi(\zeta, z) ; \zeta-z>\neq 0\right.$ for each $(\zeta, z) \in \partial U \times U$. Then $\psi$ is called an $\mathcal{A}_{p}$-boundary distinguishing map. Consider the function:

(2) $\eta^{\psi}(\zeta, z, \lambda):=\lambda(\zeta-z)<\zeta-z ; \zeta-z>^{-1}$

$+(1-\lambda) \psi(\zeta, z)<\zeta-z ; \psi(\zeta, z)>^{-1}$

(see Formula 3.1.(vii)) and the differential forms:

(3) $\check{\phi}_{p, 0}\left({ }^{s} \tilde{\eta}^{\psi}(\zeta, z, \lambda),{ }^{s} \zeta\right):=\left[\left(\tilde{\partial}{ }_{s} \zeta,{ }^{s} z+d_{\lambda}\right){ }^{s} \tilde{\eta}^{\psi}(\zeta, z, \lambda)\right] \wedge d{ }^{s} \tilde{\zeta}$,

$\widetilde{\phi}_{p, 0}^{\prime}\left({ }^{s} \tilde{\eta}^{\psi}(\zeta, z, \lambda),{ }^{s} \zeta\right):={ }^{s} \tilde{\eta}^{\psi}(\zeta, z, \lambda) d{ }^{s} \tilde{\zeta}$

(4) $\check{\phi}_{p, u}\left({ }^{s} \tilde{\eta}^{\psi}(\zeta, z, \lambda),{ }^{s} \zeta\right):=\left(i_{2 u}\left\{\left[\left(\tilde{\partial}_{s}{ }^{s},{ }^{s} z+d_{\lambda}\right){ }^{s} \tilde{\eta}^{\psi}(\zeta, z, \lambda)\right] i_{2 u}\right\}\right) \wedge\left(i_{2 u}\left(d^{s} \zeta i_{2 u}\right)\right)$, $\tilde{\phi}_{p, u}^{\prime}\left({ }^{s} \tilde{\eta}^{\psi}(\zeta, z, \lambda),{ }^{s} \zeta\right):=\left[i_{2 u}\left({ }^{s} \tilde{\eta}^{\psi}(\zeta, z, \lambda) i_{2 u}\right)\right]\left(i_{2 u}\left(d^{s} \zeta i_{2 u}\right)\right)$ for each $u>0$,

$$
\check{\phi}_{p, 0}\left({ }^{s} \tilde{\eta}^{\psi}(\zeta, z, 0),{ }^{s} \zeta\right):=\left[\tilde{\partial}{ }^{s} \zeta,{ }^{s} z{ }^{s} \tilde{\eta}^{\psi}(\zeta, z, 0)\right] \wedge d{ }^{s} \tilde{\zeta}
$$




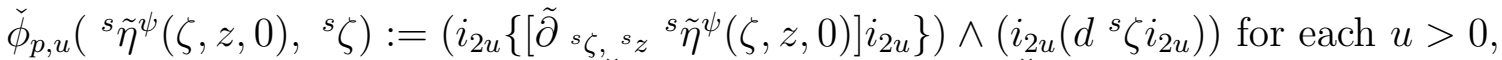

(6) $\check{w}_{2^{p}}\left({ }^{s} \tilde{\eta}^{\psi}(\zeta, z, \lambda),{ }^{s} z\right):=C_{p}\left\{\check{\phi}_{p, 0}\left({ }^{s} \tilde{\eta}^{\psi}(\zeta, z, \lambda),{ }^{s} z\right) \wedge \check{\phi}_{p, 1}\left({ }^{s} \tilde{\eta}^{\psi}(\zeta, z, \lambda),{ }^{s} z\right) \wedge \ldots\right.$

$\left.\wedge \tilde{\phi}_{p, 2^{p-1}-1}\left({ }^{s} \tilde{\eta}^{\psi}(\zeta, z, \lambda),{ }^{s} z\right)\right\}_{q_{0}\left(2^{p-1}\right)}$,

where $C_{p}=\left(\left[\left(2^{p}-2\right) !\right] 2\left(2^{p-1}-1\right)\right)^{-1}$;

(7) $\check{w}_{2^{p}, u}\left({ }^{s} \tilde{\eta}^{\psi}(\zeta, z, \lambda),{ }^{s} z\right):=\left\{\check{\phi}_{p, 0}\left({ }^{s} \tilde{\eta}^{\psi}(\zeta, z, \lambda),{ }^{s} z\right) \wedge \ldots \wedge \check{\phi}_{p, u-1}\left({ }^{s} \tilde{\eta}^{\psi}(\zeta, z, \lambda),{ }^{s} z\right) \wedge\right.$ $\left.\check{\phi}_{p, u}^{\prime}\left({ }^{s} \tilde{\eta}^{\psi}(\zeta, z, \lambda),{ }^{s} z\right) \wedge \check{\phi}_{p, u+1}\left({ }^{s} \tilde{\eta}^{\psi}(\zeta, z, \lambda),{ }^{s} z\right) \wedge \ldots \wedge \check{\phi}_{p, 2^{p-1}-1}\left({ }^{s} \tilde{\eta}^{\psi}(\zeta, z, \lambda),{ }^{s} z\right)\right\}_{q_{0}\left(2^{p-1}\right)}$, analogously to $(3-7)$ there are defined $\check{\phi}_{p, u}\left({ }^{s} \tilde{\psi}(\zeta, z)\right), \check{\phi}_{p, u}^{\prime}\left({ }^{s} \tilde{\psi}(\zeta, z)\right), \check{w}_{2^{p}}\left({ }^{s} \tilde{\psi}(\zeta, z),{ }^{s} z\right)$, $\check{w}_{2^{p}, u}\left({ }^{s} \tilde{\psi}(\zeta, z),{ }^{s} z\right)$ for each $u \geq 0$ with ${ }^{s} \tilde{\psi}(\zeta, z)$ instead of ${ }^{s} \tilde{\eta}^{\psi}(\zeta, z, \lambda)$;

$$
\begin{gathered}
(8) \phi_{\zeta, z}:=\phi_{\zeta, z}(\psi(\zeta, z) ; \zeta):=C^{\prime}{ }_{p}<\psi(\zeta, z) ; \zeta-z>^{-2^{p-1} n} \\
\sum_{s=1}^{n} \sum_{u=0}^{2^{p-1}-1}\left\{\check{w}_{2^{p}}\left({ }^{1} \tilde{\psi}(\zeta, z),{ }^{1} z\right) \wedge \ldots \wedge \check{w}_{2^{p}}\left({ }^{s-1} \tilde{\psi}(\zeta, z),{ }^{s-1} z\right) \wedge\right. \\
\left.\check{w}_{2^{p}, u}\left({ }^{s} \tilde{\psi}(\zeta, z),{ }^{s} z\right) \wedge \check{w}_{2^{p}}\left({ }^{s+1} \tilde{\psi}(\zeta, z),{ }^{s+1} z\right) \wedge \ldots \wedge \check{w}_{2^{p}}\left({ }^{n} \tilde{\psi}(\zeta, z),{ }^{n} \zeta\right)\right\}_{q_{0}(n)} ; \\
(9) \bar{\phi}_{\zeta, z, \lambda}:=\bar{\phi}_{\zeta, z, \lambda}(\psi(\zeta, z) ; \zeta):= \\
C^{\prime}{ }_{p} \sum_{s=1}^{n} \sum_{u=0}^{2^{p-1}-1}\left\{\check{w}_{2^{p}}\left({ }^{1} \tilde{\eta}^{\psi}(\zeta, z, \lambda),{ }^{1} z\right) \wedge \ldots \wedge \check{w}_{2^{p}}\left({ }^{s-1} \tilde{\eta}^{\psi}(\zeta, z, \lambda),{ }^{s-1} z\right) \wedge\right. \\
\left.\check{w}_{2^{p}, u}\left({ }^{s} \tilde{\eta}^{\psi}(\zeta, z, \lambda),{ }^{s} z\right) \wedge \check{w}_{2^{p}}\left({ }^{s+1} \tilde{\eta}^{\psi}(\zeta, z, \lambda),{ }^{s+1} z\right) \wedge \ldots \wedge \check{w}_{2^{p}}\left({ }^{n} \tilde{\eta}^{\psi}(\zeta, z, \lambda),{ }^{n} \zeta\right)\right\}_{q_{0}(n)} .
\end{gathered}
$$

If $f$ is a bounded differential form on $U$, then define the integral operators:

$$
\left(L_{\partial U}^{\psi} f\right)(z):=\int_{\zeta \in \partial U} f(\zeta) \wedge \phi_{\zeta, z}(\psi(\zeta, z) ; \zeta)
$$

$$
\left(R_{\partial U}^{\psi} f\right)(z):=\int_{\zeta \in \partial U, 0 \leq \lambda \leq 1} f(\zeta) \wedge \bar{\phi}_{\zeta, z, \lambda}(\psi(\zeta, z) ; \zeta) .
$$

3.5. Theorem. Let $U$ be an open subset in $\mathcal{A}_{p}^{n}, 2 \leq p \in \mathbf{N}$, with a piecewise $C^{1}$-boundary and let $\psi$ be an $\mathcal{A}_{p}$-boundary distinguishing map for $U$. Suppose that $f$ is a continuous mapping $f: \operatorname{cl}(U) \rightarrow \mathcal{A}_{p}$ such that $\tilde{\partial} f$ is also continuous on $U$ in the sence of distributions and has a continuous extension on $\operatorname{cl}(U)$. Then

$$
\text { (1) } f=\left(L_{\partial U}^{\psi} f\right)-\left(R_{\partial U}^{\psi} \tilde{\partial} f\right)-\left(B_{U} \tilde{\partial} f\right) \text { on } U \text {, }
$$

where the $\mathcal{A}_{p}$ integral operators $B_{U}, L_{\partial U}^{\psi}$ and $R_{\partial U}^{\psi}$ are given by Equations $3.1(13), 3.4(10,11)$.

Proof. There is the decomposition:

(2) $\bar{\phi}_{\zeta, z, \lambda}=\sum_{q=0}^{2^{p-1} n-1} \Upsilon_{q}^{\psi}(\zeta, z, \lambda)$,

where $\Upsilon_{q}^{\psi}(\zeta, z, \lambda)$ is a differential form with all terms of degree $q$ by $z$ and $\tilde{z}$ and their multiples on $\mathcal{A}_{p}$ constants and of degree $\left(2^{p} n-q-1\right)$ by $(\zeta, \lambda)$ (including $\tilde{\zeta}$ and multiples of $\zeta$ and $\tilde{\zeta}$ on $\mathcal{A}_{p}$ constants). A differential form $f$ has Decomposition 3.2(12). If $\psi(z)$ is an $\mathcal{A}_{p} z$-superdifferentiable nonzero function on an open set $V$ in $\mathcal{A}_{p}^{n}$, then differentiating the equality $(\psi(z))(\psi(z))^{-1}=e$ gives $(\psi(z))\left\{d_{z}(\psi(z))^{-1} \cdot h\right\}=-\left(d_{z} \psi(z) \cdot h\right)(\psi(z))^{-1}$ for each $z \in V$ and each $h \in \mathcal{A}_{p}^{n}$. Then $\int_{\zeta \in \partial U, 0 \leq \lambda \leq 1} f_{r}(\zeta) \wedge \Upsilon_{q}^{\psi}(\zeta, z, \lambda)=0$ for each $r \neq q+1$ since $\operatorname{dim}(\partial U)=2^{p} n-1, d \lambda \wedge d \lambda=0$ and $d \lambda$ commutes with each $b \in \mathcal{A}_{p}$. Therefore,

(3) $R_{\partial U}^{\psi} f_{r}=\int_{\zeta \in \partial U, 0 \leq \lambda \leq 1} f_{r}(\zeta) \wedge \Upsilon_{r-1}^{\psi}(\zeta, z, \lambda)$ for each $1 \leq r \leq 2^{p-1} n$ and $R_{\partial U}^{\psi} f_{r}=0$ for $r=0$ or $r>2^{p-1} n$. In particular, if $f=f_{1}$, then

(4) $R_{\partial U}^{\psi} f_{1}=\int_{\zeta \in \partial U, 0 \leq \lambda \leq 1} f_{1}(\zeta) \wedge \bar{\phi}_{\zeta, \lambda}(\psi(\zeta, z) ; \zeta)$,

where $\bar{\phi}_{\zeta, \lambda}(\psi(\zeta, z) ; \zeta)$, is obtained from $\bar{\phi}_{\zeta, z, \lambda}(\psi(\zeta, z) ; \zeta)$ by substituting all $\tilde{\partial}_{{ }} \zeta,{ }^{s} z$ in Formulas 
$3.4(3-7,9,11)$ on $\tilde{\partial}_{s_{\zeta}}$. On the other hand, with the help of Formulas $3.2(1-9,15)$ each $\mathcal{A}_{p}$ external derivative $\tilde{\partial}{ }_{s \zeta}$ can be replaced on $d_{s_{\zeta}}$ in $\bar{\phi}_{\zeta, \lambda}(\psi(\zeta, z) ; \zeta)$ in Formula (4). For $\phi_{\zeta, z}$ there is the decomposition:

(5) $\phi_{\zeta, z}=\sum_{q=0}^{2^{p-1} n-1} \Upsilon_{q}^{\psi}(\zeta, z)$, where $\Upsilon_{q}^{\psi}(\zeta, z)$ is a differential form with all terms of degree $q$ by $z$ and $\tilde{z}$ and their multiples on $\mathcal{A}_{p}$ constants and of degree $2^{p} n-q-1$ by $\zeta$ and $\tilde{\zeta}$ and their multiples on $\mathcal{A}_{p}$ constants. Therefore,

(6) $L_{\partial U}^{\psi} f_{r}=\int_{\zeta \in \partial U} f_{r} \wedge \Upsilon_{r}^{\psi}(\zeta, z)$ for each $0 \leq r \leq 2^{p-1} n-1$

and $L_{\partial U}^{\psi} f_{r}=0$ for $r \geq 2^{p-1} n$. In particular, for $f=f_{0}$ :

(7) $L_{\partial U}^{\psi} f_{0}=\int_{\zeta \in \partial U} f_{0}(\zeta) \phi_{\zeta}(\psi(\zeta, z) ; \zeta)$,

where $\phi_{\zeta}(\psi(\zeta, z))$, is obtained from $\phi_{\zeta, z}(\psi(\zeta, z) ; \zeta)$ by substituting all $\tilde{\partial}_{\zeta, z}$ in Formulas 3.4.(310) on $\partial_{\zeta}$.

In view of Formula 3.2(1) it remains to prove, that $R_{\partial U}^{\psi} \tilde{\partial} f=L_{\partial U}^{\psi} f-B_{\partial U} f$ on $U$. For each $\zeta$ in a neighborhood of $\partial U$ there is the identity:

(8) $<\eta^{\psi}(\zeta, z, \lambda) ; \zeta-z>=1$ for each $0 \leq \lambda \leq 1$, hence $d_{\zeta, z, \lambda}\left\langle\eta^{\psi}(\zeta, z, \lambda) ; \zeta-z>=0\right.$. By Formulas 3.2(15-18) :

(9) $d_{\zeta, \lambda} \bar{\phi}_{\zeta, z, \lambda}=0$. From Identities $3.2(15-18)$ it follows, that

(10) $\partial_{\zeta} f \wedge \bar{\phi}_{\zeta, \lambda}=0$. Therefore, from (4), (9), (10) it follows, that

(11) $d_{\zeta, \lambda}\left[f(\zeta) \bar{\phi}_{\zeta, \lambda}\right]=\left[\tilde{\partial}_{\zeta} f(\zeta)\right] \wedge \bar{\phi}_{\zeta, \lambda}$, since $\tilde{\partial}_{\zeta} f(\zeta)=\sum_{s=1}^{n}\left(\partial f(\zeta, \tilde{\zeta}) / \partial{ }^{s} \tilde{\zeta}\right) \cdot d^{s} \tilde{\zeta}$. Due to Formulas 3.2(15-17) and 3.4(1-9):

(12) $\left.\bar{\phi}_{\zeta, \lambda}\right|_{\lambda=0}=\phi_{\zeta},\left.\bar{\phi}_{\zeta, \lambda}\right|_{\lambda=1}=\theta_{z}(\zeta)$.

If $\Upsilon(\zeta, z, \lambda)$ is a differential form over $\mathcal{A}_{p}$, then

$\Upsilon(\zeta, z, \lambda)=\sum_{s=0}^{2^{p}-1} \Psi_{s}\left(\zeta_{0}, \ldots, \zeta_{2^{p}-1}, z_{0}, \ldots, z_{2^{p}-1}, \lambda\right) i_{s}$, where $\zeta=\zeta_{0} i_{0}+\ldots+\zeta_{2^{p}-1} i_{2^{p}-1}, \zeta, z \in \mathcal{A}_{p}, \zeta_{0}, \ldots, \zeta_{2^{p}-1}, z_{0}, \ldots, z_{2^{p}-1}, \lambda \in \mathbf{R},\left\{i_{0}, \ldots, i_{2^{p}-1}\right\}$ denotes the set of standard generators of $\mathcal{A}_{p}, \Psi_{s}$ is with values in $\mathbf{R}$ for each $s=0, \ldots, 2^{p}-1$. From the Stoke's formula for vector-valued differential forms, in particular, for $\left[f(\zeta) \bar{\phi}_{\zeta, z, \lambda}(\psi(\zeta, z) ; \zeta)\right]$ on $\partial U \times[0,1]$ and Formulas $(4),(7),(11),(12)$ above it follows the statement of this theorem.

3.6. Corollary. Let conditions of Theorem 3.5 be satisfied and let $f$ be an $\mathcal{A}_{p}$ holomorphic function on $U$, then $f=L_{\partial U}^{\psi} f$ on $U$.

3.7. Remark. For $n=1$ Formula 3.2.(1) produces another analog of the Cauchy-Green formula (see Theorem 2.1 and Remark 2.1.1) without using the $\mathcal{A}_{p}$ line integrals. This is caused by the fact that the dimension of $\mathcal{A}_{p}$ over $\mathbf{R}$ is greater, than 2: $\operatorname{dim}_{\mathbf{R}} \mathcal{A}_{p}=2^{p}$, that produces new integral relations. Theorem 3.2 can be used instead of Theorem 2.1 to prove theorems 2.3 and 2.11 (with differential forms of Theorem 3.2 instead of differential forms of Theorem 2.1).

If $\psi(\zeta, z)=\zeta-z$, then $L_{\partial U}^{\psi}=B_{\partial U}$ and $R_{\partial U}^{\psi}=0$, hence Formula 3.5.(1) reduces to Formula 3.2.(1). For a function $f$ or a 1 -form $f$ Formulas 3.2 $(13,14)$ respectively are valid as well for $\bar{\theta}(\zeta, z)$ instead of $\theta_{z}(\zeta)$, where $d_{\zeta, z} \bar{\theta}(\zeta, z)=0$ for each $\zeta \neq z$. A choice of $\phi_{p, s}$ and $w_{2^{p}}$ is not unique, for example, $d \tilde{\zeta} \wedge d \zeta \wedge d \zeta \wedge d \zeta$ may be taken in $\mathbf{H}$, since it gives up to a multiplier $C e$, where $C$ is a real constant, the canonical volume element in $\mathbf{H}$ and $d \zeta \wedge d \zeta \wedge d \zeta \wedge d \zeta=0$

Formulas 3.2.(1) and 3.5.(1) for functions of $\mathcal{A}_{p}$ variables are the $\mathcal{A}_{p}$ analogs of the Martinelli-Bochner and the Leray formulas for functions of complex variables respectively, where $\psi(\zeta, z)$ is the $\mathcal{A}_{p}$ analog of the Leray complex map (see $\S 3.4$ ). In the $\mathcal{A}_{p}$ case the algebra of differential forms bears the additional gradation structure and have another properties, than in the complex case (see also $\S \S 2.8$ and $3.7[19]$ ). Lemma 3.9 below shows, that the $\mathcal{A}_{p}$ boundary distinguishing maps exist.

3.8. Definitions and Notations. Let a subset $U$ in $\mathcal{A}_{p}^{n}, 2 \leq p \in \mathbf{N}$ or $p=\Lambda$, be given 
by the equation:

(1) $U:=\left\{z \in \mathcal{A}_{p}^{n}: \rho(z)<0\right\}$, where $\rho$ is a real-valued $C^{2}$-function such that there exists a constant $\epsilon_{0}>0$ for which:

(2) $\sum_{l, m=1}^{2^{p} n}\left(\partial^{2} \rho(z) / \partial x_{l} \partial x_{m}\right) t_{l} t_{m} \geq \epsilon_{0}|t|^{2}$ for each $t \in \mathbf{R}^{\mathbf{2}^{\mathbf{p}} \mathbf{n}}$ for finite $p$ with ${ }^{l} z=\sum_{m=1}^{2^{p}} x_{2^{p}(l-1)+m} S_{m}, S_{m}:=i_{m-1}$ for each $m, x_{l} \in \mathbf{R}$; or

$(2)^{\prime} \sum_{l, m=1}^{n}\left(\partial^{2} \rho(z) / \partial^{l} z \partial^{m} \tilde{z}\right) .\left({ }^{l} h,{ }^{m} \tilde{h}\right) \geq \epsilon_{0}\|h\|^{2}$ for each $h \in \mathcal{A}_{p}^{n}$ for infinite $p=\Lambda$, where $z=\left({ }^{1} z, \ldots,{ }^{n} z\right),{ }^{l} z \in \mathcal{A}_{p}$. Then $U$ is called strictly convex open subset (with $C^{2}$-boundary). Let

(3) $w_{\rho}(z):=\left(\partial \rho(z) / \partial^{1} z, \ldots, \partial \rho(z) / \partial^{n} z\right)$.

Put for finite $2 \leq p \in \mathbf{N}$

$v_{\rho}(z):=\sum_{m=1}^{2^{p}}\left(w_{\rho} . S_{m}\right) S_{m}$, where as usually $w_{\rho} \cdot S_{m}=\left(d_{z} \rho(z)\right) \cdot S_{m}$ is the differential of the function $\rho$.

For infinite $p=\Lambda$ take a collar neighborhood $V$ for $\partial U$ such that for each $\zeta \in V$ there exists a unique point $\xi \in \partial U$, for which $\zeta$ belongs to the segment of the straight line intersecting $\partial U$ at the point $\xi$ along an outside normal (perpendicular) vector $n_{\xi}$ to $\partial U$ at the point $\xi \in \partial U, \xi=\xi(\zeta)$. Put

$<v_{\rho}(\zeta) ; h>:=\sum_{m}<\left((\partial \rho(\zeta) / \partial \zeta) . S_{m}\right) S_{m} ; h>$,

where $h \in \mathcal{A}_{p}^{n}$, that defines $v_{\rho}$, since $\|h\|<\infty$ for each $h \in \mathcal{A}_{p}^{n}$ and $(\partial \rho(\zeta) / \partial \zeta) \in C^{1}$ is a bounded operator for each $\zeta$.

3.9. Lemma. Let the function $v_{\rho}$ be as in $\S 3.8$. Then $v_{\rho}$ is the $\mathcal{A}_{p}$-boundary distinguishing map for $U$.

Proof. Since $S_{m} S_{l}=(-1)^{\kappa\left(S_{m}\right) \kappa\left(S_{l}\right)} S_{l} S_{m}$ for each $m \neq l$, where $\kappa\left(S_{1}\right)=0, \kappa\left(S_{m}\right)=1$ for each $m>1$, then

$<v_{\rho}(\zeta) ; \zeta-z>+<\zeta-z ; v_{\rho}(\zeta)>=2 \sum_{l=1}^{2^{p} n}\left(\partial \rho(\zeta) / \partial x_{l}\right) x_{l}(\zeta-z)$,

when $p$ is finite, where $x_{l}=x_{l}(\zeta)$ and $x_{l}(\zeta-z)$ are real coordinates corresponding to $\zeta$ and $\zeta-z$. For infinite $p=\Lambda$ there is the equality:

$<v_{\rho}(\zeta) ; \zeta-z>+<\zeta-z ; v_{\rho}(\zeta)>=2 \operatorname{Re}\left[\sum_{l=1}^{n}\left(\partial \rho(\zeta) / \partial^{l} \zeta\right) \cdot\left({ }^{l} \zeta-{ }^{l} z\right)\right]$.

By the Taylor's theorem: $\rho(z)=\rho(\zeta)-<v_{\rho}(\zeta) ; \zeta-z>/ 2-<\zeta-z ; v_{\rho}(\zeta)>/ 2+$ $\sum_{l, m=1}^{n}\left(\partial^{2} \rho(\zeta) / \partial^{l} \zeta \partial^{m} \zeta\right) \cdot\left(\left({ }^{l} \zeta-{ }^{l} z\right),\left({ }^{m} \zeta-{ }^{m} z\right) / 2+o\left(|\zeta-z|^{2}\right)\right.$. Therefore, there exists a neighborhood $V$ of $\partial U$ and $\epsilon_{1}>0$ such that

(1) $\left(<v_{\rho}(\zeta) ; \zeta-z>+<\zeta-z ; v_{\rho}(\zeta)>\right) / 2 \geq \rho(\zeta)-\rho(z)+\epsilon_{0}|\zeta-z|^{2} / 4$ for each $\zeta \in V$ and $|\zeta-z| \leq \epsilon_{1}$, where $a=\sum_{m} a_{m} S_{m}$ for each $a \in \mathcal{A}_{p}, a_{m}$ are reals. If $z \in U, \zeta \in \partial U$, $|z-\zeta| \leq \epsilon_{1}$, then by $(1):\left(\left\langle v_{\rho}(\zeta) ; \zeta-z>+\left\langle\zeta-z ; v_{\rho}(\zeta)>\right) \geq-\rho(z)>0\right.\right.$. If $|\zeta-z|>\epsilon_{1}$, put $z_{1}:=\left(1-\epsilon_{1}|\zeta-z|^{-1}\right) \zeta+\epsilon_{1}|\zeta-z|^{-1} z$, then $\zeta-z_{1}=\epsilon_{1}|\zeta-z|^{-1}(\zeta-z)$, consequently, $\left(<v_{\rho}(\zeta) ; \zeta-z>+<\zeta-z ; v_{\rho}(\zeta)>\right)_{e} / 2 \geq-\rho\left(z_{1}\right)$. Evidently, $U$ is convex and $z_{1} \in U$.

3.10. Theorem. Let $U$ be a strictly convex open subset in $\mathcal{A}_{p}^{n}, 2 \leq p \in \mathbf{N}$ or $p=\Lambda$, (see 3.8.(1)) and let $f$ be a continuous function on $U$ with continuous $\tilde{\partial} f$ on $U$ in the sense of distributions having a continuous extension on $c l(U)$ such that 2.11. $(i)$ is satisfied. Then there exists a function $u$ on $U$ which is a solution of the $\tilde{\partial}$-equation 2.11.(ii).

Proof. Reduce the proof to the finite case as in $§ 2.3$. In proofs of Theorems 2.3 and 2.11 take in Formula 3.5(1) $\chi f$ instead of $f$, which is possible due to Lemma 3.9, choosing $\psi=v_{\rho}$ and $\operatorname{supp}(\chi)$ as a proper subset of $U$. Then $L_{\partial U}^{\psi} \chi f=0$ and $R_{\partial U}^{\psi} \chi f=0$, hence $\chi f=$ $-B_{U} \partial \chi f$. For each fixed $z \in U$ a subset ${ }^{l} U_{\eta}:=\left\{\xi \in \mathcal{A}_{p}: \rho\left({ }^{1} z, \ldots,{ }^{l-1} z, \xi,{ }^{l+1} z, \ldots,{ }^{n} z\right)<\right.$ $0\}$ is strictly convex in $\mathcal{A}_{p}$ due to $3.8(1,2)$, where $\eta:=\left({ }^{1} z, \ldots,{ }^{l-1} z,{ }^{l+1} z, \ldots,{ }^{n} z\right)$. Apply $3.5(1)$ by a variable $\xi$ in ${ }^{l} U_{\eta}$, in particular, for $l=1$, for which $v_{\rho}$ by the variable $\xi$ is the $\mathcal{A}_{p}$-boundary distinguishing map for ${ }^{1} U_{\eta}$. Therefore, $u(z):=-B{ }^{1} U_{\eta}{ }^{1} \hat{f}(\xi, \eta) . d \tilde{\xi}$ with $z=(\xi, \eta), \xi \in{ }^{1} U_{\eta}$ solves the problem. 


\section{Manifolds over Cayley-Dickson algebras}

4.1. Definitions and Notations. Suppose that $M$ is an $\mathcal{A}_{p}$ manifold and let $R L\left(N, \mathcal{A}_{p}\right)$ be the family of all right $\mathcal{A}_{p}$-superlinear operators $A: \mathcal{A}_{p}^{N} \rightarrow \mathcal{A}_{p}^{N}$, where $2 \leq p \in \mathbf{N}$ or $p=\Lambda$. Then an $\mathcal{A}_{p}$ holomorphic vector bundle $Q$ of $\mathcal{A}_{p}$ dimension $N$ over $M$ is a $C^{\infty}$-vector bundle $Q$ over $M$ with the characteristic fibre $\mathcal{A}_{p}^{N}$ together with an $\mathcal{A}_{p}$ holomorphic atlas of local trivializations: $g_{a, b}: U_{a} \cap U_{b} \rightarrow R L\left(N, \mathcal{A}_{p}\right)$, where $U_{a} \cap U_{b} \neq \emptyset,\left\{\left(U_{a}, h_{a}\right): a \in \Upsilon\right\}=\operatorname{At}(Q)$, $\bigcup_{a} U_{a}=M, U_{a}$ is open in $M, h_{a}:\left.Q\right|_{U_{a}} \rightarrow U_{a} \times \mathcal{A}_{p}^{N}$ is the bundle isomorphism, $\left(z, g_{a, b}(z) v\right)=$ $h_{a} \circ h_{b}^{-1}(z, v), z \in U_{a} \cap U_{b}, v \in \mathcal{A}_{p}^{N}$. If $Y_{N}$ is the underlying to $\mathcal{A}_{p}^{N}$ real vector space, then suppose, that each $g_{a, b}$ induces a R-linear isomorphism of $Y_{N}$ onto itself. Since $M$ has the real underlying manifold $M_{\mathbf{R}}$, then there exists the tangent bundle $T M$ such that $T_{x} M$ is isomorphic with $\mathcal{A}_{p}^{n}$ for each $x \in M$, since $T U_{a}=U_{a} \times \mathcal{A}_{p}^{n}$ for each $a$, where $\operatorname{dim}_{\mathcal{A}_{p}} M=n$ is the $\mathcal{A}_{p}$ dimension of $M$.

If $X$ is a Banach space over $\mathcal{A}_{p}$ (with left and right distributivity laws relative to multiplications of vectors in $X$ on scalars from $\mathcal{A}_{p}$ ), then denote by $X_{q}^{*}$ the space of all additive $\mathbf{R}$-homogeneous functionals on $X$ with values in $\mathcal{A}_{p}$. Clearly $X_{q}^{*}$ is the Banach space over $\mathcal{A}_{p}$. Then $T^{*} M$ with fibres $\left(\left(\mathcal{A}_{p}^{n}\right)_{q}\right)^{*}$ denotes the $\mathcal{A}_{p}$ cotangent bundle of $M$ and $\Lambda^{r} T^{*} M$ denotes the vector bundle whose sections are $\mathcal{A}_{p}$ r-forms on $M$, where $S_{b} d x_{b} \wedge S_{a} d x_{a}=$ $-(-1)^{\kappa\left(S_{a}\right) \kappa\left(S_{b}\right)} S_{a} d x_{a} \wedge S_{b} d x_{b}$ for each $S_{a} \neq S_{b} \in\left\{i_{0}, \ldots, i_{2^{p}-1}\right\}, d z=\sum_{m=1}^{2^{p}} d x_{m} S_{m}, z \in \mathcal{A}_{p}$, $x_{b} \in \mathbf{R}$.

The $\mathcal{A}_{p}$ holomorphic Cousin data in $Q$ is a family $\left\{f_{a, b}: a, b \in \Upsilon\right\}$ of $\mathcal{A}_{p}$ holomorphic sections $f_{a, b}: U_{a} \cap U_{b} \rightarrow Q$ such that $f_{a, b}+f_{b, l}=f_{a, l}$ in $U_{a} \cap U_{b} \cap U_{l}$ for each $a, b, l \in \Upsilon$. A finding of a family $\left\{f_{a}: a \in \Upsilon\right\}$ of $\mathcal{A}_{p}$ holomorphic sections $f_{a}: U_{a} \rightarrow Q$ such that $f_{a, b}=f_{a}-f_{b}$ in $U_{a} \cap U_{b}$ for each $a, b \in \Upsilon$ will be called the $\mathcal{A}_{p}$ Cousin problem.

4.2. Theorem. Let $M$ be an $\mathcal{A}_{p}$ manifold and $Q$ be an $\mathcal{A}_{p}$ holomorphic vector bundle on $M$, where $2 \leq p \in \mathbf{N}$ or $p=\Lambda$. Then Conditions $(i, i i)$ are equivalent:

(i) each $\mathcal{A}_{p}$ holomorphic Cousin problem in $M$ has a solution;

(ii) for each $\mathcal{A}_{p}$ holomorphic section $f$ of $Q$ such that $\tilde{\partial} f=0$ on $M$, there exists a $C^{\infty}$-section $U$ of $Q$ such that $(\partial u / \partial \tilde{z})=\hat{f}$ on $M$.

Proof. $(i) \rightarrow(i i)$. In view of Theorems 2.11 and 3.10 there exists an (open) covering $\left\{U_{a}: a\right\}$ of $M$ and $C^{\infty}$-sections $u_{b}: U_{b} \rightarrow Q$ such that $\left(\partial u_{b} / \partial \tilde{z}\right)=\hat{f}$ in $U_{b}$. Then $\left(u_{b}-u_{l}\right)$ is $\mathcal{A}_{p}$ holomorphic in $U_{b} \cap U_{l}$ and their family forms the $\mathcal{A}_{p}$ holomorphic Cousin data in $Q$. Put $u:=u_{b}-h_{b}$ on $U_{b}$, where $u_{b}-u_{l}=h_{b}-h_{l}, h_{b}: U_{b} \rightarrow Q$ is an $\mathcal{A}_{p}$ holomorphic section given by $(i)$.

$(i i) \rightarrow(i)$. Take a $C^{\infty}$-partition of unity $\left\{\chi_{b}: b\right\}$ subordinated to $\left\{U_{b}: b\right\}$ and $c_{b}:=$ $-\sum_{a} \chi_{a} f_{a, b}$ on $U_{b}$, then $f_{l, b}=\sum_{a} \chi_{a}\left(f_{l, a}+f_{a, b}\right)=c_{l}-c_{b}$ in $U_{l} \cap U_{b}$, hence $\left(\partial c_{l} / \partial \tilde{z}\right)=\left(\partial c_{b} / \partial \tilde{z}\right)$ in $U_{l} \cap U_{b}$. By $(i i)$ there exists a $C^{\infty}$-section $u: M \rightarrow Q$ with $(\partial u / \partial \tilde{z})=\left(\partial c_{b} / \partial \tilde{z}\right)$ on $U_{b}$ and $h_{b}:=c_{b}-u$ on $U_{b}$ gives the solution.

4.3. Definitions. Suppose $U$ is an open subset in $\mathcal{A}_{p}$, then a $C^{2}$-function $\rho: U \rightarrow \mathbf{R}$ is called subharmonic (strictly subharmonic) in $U$ if $\sum_{m=1}^{2^{p}} \partial^{2} \rho / \partial x_{m}^{2} \geq 0\left(\sum_{m=1}^{2^{p}} \partial^{2} \rho / \partial x_{m}^{2}>0\right.$ correspondingly) for finite $p \geq 2$; or

$\left(\partial^{2} \rho(z) / \partial z \partial \tilde{z}\right) \cdot(\xi, \tilde{\xi}) \geq 0$ (or $>0$ ) for each $z \in U$ and each $0 \neq \xi \in \mathcal{A}_{p}$ for $p=\Lambda$, where $z=\sum_{m=1}^{2^{p}} x_{m} S_{m} \in U$, where $x_{m} \in \mathbf{R}$ for each $m$.

If $U$ is an open subset in $\mathcal{A}_{p}^{n}$, then a $C^{2}$-function $\rho: U \rightarrow \mathbf{R}$ such that the function $\zeta \mapsto \rho(v+\zeta w)$ is subharmonic (strictly subharmonic) on its domain for each $v, w \in \mathcal{A}_{p}^{n}$ is called plurisubharmonic (strictly plurisubharmonic correspondingly) function, where $\zeta \in \mathcal{A}_{p}$.

A $C^{v}$-function $\rho$ on an $\mathcal{A}_{p}$ manifold $M$ is called a strictly plurisubharmonic exhausting $C^{v}$-function for $M, 2 \leq v \in \mathbf{N}$, if $\rho$ is a strictly plurisubharmonic $C^{v}$-function on $M$ and for each $\alpha \in \mathbf{R}$ the set $\{z \in M: \rho(z)<\alpha\}$ is bounded in $M$. 
4.4. Theorem. Let $M$ be an $\mathcal{A}_{p}$ manifold with strictly plurisubharmonic exhausting function $\rho$ such that $\rho$ is a $C_{z, \tilde{z}}^{\omega}$-function and let $Q$ be an $\mathcal{A}_{p}$ holomorphic vector bundle on $M, U_{\alpha}:=\{z \in M: \rho(z)<\alpha\}$ for $\alpha \in \mathbf{R}$, where $2 \leq p \in \mathbf{N}$ or $p=\Lambda$.

(i). Suppose that $d \rho(z) \neq 0$ for each $z \in \partial U_{\alpha}$ for a marked $\alpha \in \mathbf{R}$. Then every continuous section $f: \operatorname{cl}\left(U_{\alpha}\right) \rightarrow Q$ which is $\mathcal{A}_{p}$ holomorphic on $U_{\alpha}$ can be approximated (uniformly for finite $p)$ on $\mathrm{cl}\left(U_{\alpha}\right)$ by $\mathcal{A}_{p}$ holomorphic sections of $Q$ on $M$.

(ii). For each continuous mapping $f: M \rightarrow Q$ such that $\tilde{\partial} f=0$ on $M$ there exists a continuous mapping $u: M \rightarrow Q$ such that $\partial u / \partial \tilde{z}=\hat{f}$ on $M$.

Proof. For a $C_{z, \tilde{z}}^{\omega}$-function $\rho: U \rightarrow \mathbf{R}$ (that is, $\rho$ is locally analytic in variables $(z, \tilde{z})$, $\left.\mathbf{R}=\mathbf{R} e \hookrightarrow \mathcal{A}_{p}\right)$ there is the identity:

$\sum_{l, m, a, b}\left(\partial^{2} \rho / \partial^{l} x_{a} \partial^{m} x_{b}\right) t_{2^{p}(l-1)+a} t_{2^{p}(l-1)+b}=$ $\sum_{m, l}\left(\partial^{2} \rho(z) / \partial^{l} z \partial^{m} z\right) \cdot\left(\left(\partial^{l} z / \partial^{l} x_{a}\right) t_{2^{p}(l-1)+a},\left(\partial^{m} z / \partial^{l} x_{b}\right) t_{2^{p}(l-1)+b}\right)$ $=\sum_{m, l=1}^{n}\left(\partial^{2} \rho(z) / \partial^{l} z \partial^{m} \tilde{z}\right) \cdot\left({ }^{l} \xi,{ }^{m} \tilde{\xi}\right)$ for finite $p$, since $\partial^{l} z / \partial^{l} x_{a}=S_{a}, \partial^{l} \tilde{z} / \partial{ }^{l} x_{a}=(-1)^{\kappa\left(S_{a}\right)} S_{a}$, where ${ }^{l} \xi=\sum_{m=1}^{2^{p}} t_{2^{p}(l-1)+m} S_{m}, S_{m}=i_{m-1}$ for each $m,{ }^{l} z=\sum_{m=1}^{2^{p}}{ }^{l} x_{m} S_{m}, t_{b} \in \mathbf{R},{ }^{l} x_{m} \in \mathbf{R}$. Therefore, a $C_{z, \tilde{z}}^{\omega}$-function $\rho$ is strictly plurisubharmonic on $U$ if and only if

(1) $\sum_{m, l=1}^{n}\left(\partial^{2} \rho(z) / \partial^{l} z \partial^{m} \tilde{z}\right) .\left({ }^{l} \xi,{ }^{m} \tilde{\xi}\right)>0$ for each $z \in U$ and each $0 \neq \xi \in \mathcal{A}_{p}^{n}$, where $\xi=\left({ }^{1} \xi, \ldots,{ }^{n} \xi\right)$ (see also $\left.\S 2[19,20]\right)$. Consider a proper bounded closed subset $A$ in $M$ such that $d \rho(z) \neq 0$ for each $z \in A$. Then for each $\epsilon>0$ there exists a strictly plurisubharmonic function $\rho_{\epsilon}: M \rightarrow \mathbf{R}$ such that $\rho e$ is a $C_{z, \tilde{z}}^{\omega}$-function on $M$ and $(i-i i i)$ are fulfilled:

(i) $\rho-\rho_{\epsilon}$ together with its first and second derivatives is not greater than $\epsilon$ on $M$;

(ii) the set $\operatorname{Crit}\left(\rho_{\epsilon}\right):=\left\{z \in M: d \rho_{\epsilon}(z)=0\right\}$ is discrete in $M$;

(iii) $\rho_{\epsilon}=\rho$ on $A$ (see also Lemma 2.1.2.2 [7] in the complex case).

The space $C_{z}^{\omega}\left(U, \mathcal{A}_{p}\right)$ is dense in $C^{0}\left(U, \mathcal{A}_{p}\right)$ for each open $U$ in $\mathcal{A}_{p}^{n}$ (see $\S 2.7$ and Theorem 3.28 in $[19,20])$. Suppose $\beta \in \mathbf{R}$ and $d \rho(z) \neq 0$ for $z \in \partial U_{\beta}$ and $f: \operatorname{cl}\left(U_{\beta}\right) \rightarrow Q$ is a continuous section $\mathcal{A}_{p}$ holomorphic on $U_{\beta}$. Therefore, for each $\beta \leq \alpha<\infty$ if $d \rho(z) \neq 0$ for each $z \in \partial U_{\alpha}$, then $f$ can be approximated (uniformly for finite $p$ ) on $\operatorname{cl}\left(U_{\beta}\right)$ by continuous sections on $\operatorname{cl}\left(U_{\alpha}\right)$ that are holomorphic on $U_{\alpha}$. There exists a sequence $\beta<\alpha_{1}<\alpha_{2}<\ldots$ such that $\lim _{l} \alpha_{l}=\infty$ and $d \rho(z) \neq 0$ for each $z \in \partial U_{\alpha_{l}}$, since $\operatorname{Crit}(\rho)$ is discrete. For each $\epsilon>0$ and each natural number $s$ satisfying $2 \leq s \leq p$ there exists a continuous section $f_{l}: \operatorname{cl}\left(U_{\alpha_{l}}\right) \rightarrow Q$ such that $f_{l}$ is $\mathcal{A}_{p}$ holomorphic on $U_{\alpha_{l}}$ and $\left\|f_{l+1}-f_{l}\right\|_{C^{0}\left(U_{\alpha_{l}, s}\right)}<\epsilon 2^{-l-1}$ for each $l \in \mathbf{N}$, where $f_{0}:=f, M_{s}$ denotes the $\left(2^{s}\right.$-dimensional over $\left.\mathbf{R}\right)$ closed submanifold in $M$ induced by the embedding of $\mathcal{A}_{s}$ into $\mathcal{A}_{p}$, when $p$ is infinite, or put $M_{s}=M$ for finite $p$ taking $s=p, U_{\alpha_{l}, s}:=U_{\alpha_{l}} \cap M_{s}$. Therefore, the sequence $\left\{f_{l}: l \in \mathbf{N}\right\}$ converges to the $\mathcal{A}_{s}$ holomorphic section $g: M_{s} \rightarrow Q$ uniformly on each compact subset $P$ in $M_{s}$ and $\|f-g\|_{C^{0}\left(U_{\beta, s}\right)}<\epsilon$.

The second statement $(i i)$ follows from $(i)$ and Theorems 2.11,3.10, since Crit $(\rho)$ is discrete in $M$ and there exists a sequence of continuous $Q$-valued functions on $\operatorname{cl}\left(U_{\alpha_{l}}\right)$ such that $\partial u_{l} / \partial \tilde{z}=\hat{f}$ in the sense of distributions on $U_{\alpha_{l}}, \bigcup_{l} U_{\alpha_{l}}=M$ (see also the complex case in $\S 2.12 .3$ [7] mentioning, that Lemma 2.12.4 there can be reformulated and proved for an $\mathcal{A}_{p}$ manifold $M$ on $\mathcal{A}_{p}^{n}$ instead of a complex manifold on $\mathbf{C}^{\mathbf{n}}$ ).

4.5. Definitions. Let $M$ be an $\mathcal{A}_{p}$ manifold (see $\S 2.10$ ), where $2 \leq p \in \mathbf{N}$ or $p=\Lambda$. For a closed bounded subset $G$ in $M$ put: $\hat{G}_{M}^{\mathcal{H}}:=\left\{z \in M:|f(z)| \leq \sup _{\zeta \in G}|\hat{f}(\zeta)| \quad \forall f \in \mathcal{H}(M)\right\}$. Such $\hat{G}_{M}^{\mathcal{H}}$ is called the $\mathcal{H}(M)$-hull of $G$. If $G=\hat{G}_{M}^{\mathcal{H}}$, then $G$ is called $\mathcal{H}(M)$-convex. An $\mathcal{A}_{p}$ manifold $M$ is called $\mathcal{A}_{p}$ holomorphically convex if for each closed bounded subset $G$ in $M$ the set $\hat{G}_{M}^{\mathcal{H}}$ is closed and bounded.

An $\mathcal{A}_{p}$ manifold $M$ with a countable atlas $A t(M)$ having dimension $n$ over $\mathcal{A}_{p}$ and satisfying $(i, i i)$ :

(i) $M$ is $\mathcal{A}_{p}$ holomorphically convex; 
(ii) for each $z \in M$ there are ${ }^{1} f, \ldots,{ }^{n} f \in \mathcal{H}(M)$ and there exists a neighbourhood $U$ of $z$ such that the map $U \ni \zeta \mapsto\left({ }^{1} f(\zeta), \ldots,{ }^{n} f(\zeta)\right)$ is $\mathcal{A}_{p}$ biholomorphic (see $\S 2.6$ ), then $M$ is called an $\mathcal{A}_{p}$ Stein manifold.

4.6. Remark. If $M_{1}$ and $M_{2}$ are two $\mathcal{A}_{p}$ Stein manifolds, then $M_{1} \times M_{2}$ is an $\mathcal{A}_{p}$ Stein manifold. If $N$ is a closed $\mathcal{A}_{p}$ submanifold of a $\mathcal{A}_{p}$ Stein manifold $M$, then $N$ is also an $\mathcal{A}_{p}$ Stein manifold.

4.7. Theorem. Let $M$ be an $\mathcal{A}_{p}$ Stein manifold, where $2 \leq p \in \mathbf{N}$ or $p=\Lambda$. Then for each $\mathcal{H}(M)$-convex closed bounded subset $P$ in $M, P \neq M$ and each neighborhood $V_{P}$ of $P$ there exists a strictly plurisubharmonic exhausting $C_{z, \tilde{z}}^{\omega}$ function $\rho$ on $M$ such that $\rho<0$ on $P$ and $\rho>0$ on $M \backslash V_{P}$.

The proof of this theorem is analogous to that of Theorem 2.3.14 [7] in the complex case taking $\rho(z):=-1+\sum_{l=1}^{\infty} \sum_{k=1}^{N(l)} f_{l}^{k}(z)\left(f_{l}^{k}(z)\right)^{\tilde{*}}$ for each $z \in M$, where $f_{l}^{k} \in C_{z, \tilde{z}}^{\omega}, M=$ $\bigcup_{l} P_{l}, P_{l} \subset \operatorname{Int}\left(P_{l+1}\right)$ for each $l \in \mathbf{N}$, each $P_{l}$ is $\mathcal{H}(M)$-convex, $\sum_{k=1}^{N(l)}\left|f_{l}^{k}(z)\right|^{2}<2^{-l}$ for each $z \in P_{l}, \sum_{k=1}^{N(l)}\left|f_{l}^{k}(z)\right|^{2}>l$ for each $z \in P_{l+2} \backslash U_{l}, U_{l}:=\operatorname{Int}\left(P_{l+1}\right)$, with the rank $\operatorname{rank}\left[\left(\partial f_{l}^{k} / \partial^{m} z\right)_{m=1, \ldots, n}^{k=1, \ldots, N}\right]=2^{p} n$ over $\mathbf{R}$ for each $z \in P_{l}$ for finite $p$ or $\left(f^{1}, \ldots, f^{N(l)}\right)(z)$ is regular for infinite $p$ (see Definitions 2.6 and 4.3).

4.8. Theorem. An $\mathcal{A}_{p}$ manifold $M$ is an $\mathcal{A}_{p}$ Stein manifold if and only if there exists a strictly plurisubharmonic exhausting $C_{z, \tilde{z}}^{\omega}$ function $\rho$ on $M$, then $\{z \in M: \rho(z) \leq \alpha\}$ is $\mathcal{H}(M)$-convex for each $\alpha \in \mathbf{R}$, where $2 \leq p \in \mathbf{N}$ or $p=\Lambda$.

Proof. The necessity follows from Theorem 4.7. To prove sufficiency suppose $\eta=$ $\left({ }^{1} \eta, \ldots,{ }^{n} \eta\right)$ are $\mathcal{A}_{p}$ holomorphic coordinates in a neighbourhood $V_{\xi}$ of $\xi \in M$. Consider

(1) $u(z):=2 \sum_{l, m=1}^{n}<v_{\rho}(\xi) ; \eta(z)-\eta(\xi)>$ $+\sum_{l, m=1}^{n}\left(\partial^{2} \rho(\xi) / \partial{ }^{l} \eta \partial{ }^{m} \eta\right) \cdot\left[\left({ }^{l} \eta(z)-{ }^{l} \eta(\xi)\right),\left({ }^{m} \eta(z)-{ }^{m} \eta(\xi)\right)\right] e$, where $v_{\rho}(\xi)$ is given by 3.8.(3). Then $u$ is holomorphic in $V_{\xi}$ and $u(\xi)=0$. By Lemma 3.9:

(2) $\quad(u(z)+\tilde{u}(z)) / 2=\rho(z)-\rho(\xi)-\sum_{l, m=1}^{n}\left(\partial^{2} \rho(\xi) / \partial{ }^{l} \eta \partial{ }^{m} \tilde{\eta}\right) \cdot\left[\left({ }^{l} \eta(z)-{ }^{l} \eta(\xi)\right),\left({ }^{m} \eta(z)-\right.\right.$ $\left.\left.{ }^{m} \eta(\xi)\right)^{\tilde{*}}\right]+o\left(|\eta(\xi)-\eta(z)|^{2}\right)$. From the strict plurisubharmonicity of $\rho$ it follows, that there exists $\beta>0$ and $V_{\xi}$ such that

(3) $(u(z)+\tilde{u}(z)) / 2<\rho(z)-\rho(\xi)-\beta|\eta(z)-\eta(\xi)|^{2}$ for each $z \in V_{\xi}$. Then $\exp (u(\xi))=1$ and $|\exp (u(z))|<1$ for each $\xi \neq z \in \operatorname{cl}\left(U_{\alpha}\right) \cap V_{\xi}$ (see Corollary 3.3 [19], Corollary 3.3 and Note 3.6.3 [20]).

If $g: \mathbf{R} \rightarrow \mathcal{A}_{p}$ is a $C^{\infty}$-function with bounded (closed) support, then $g(z \tilde{z})=: \chi(z)$ is a $C^{\infty}$-function on $\mathcal{A}_{p}^{n}$ with bounded closed support such that $\chi$ is $\mathcal{A}_{p}(z, \tilde{z})$-superdifferentiable. Therefore, there exists a neighbourhood $W_{\xi} \subset V_{\xi}$ of $\xi$ and an infinitely $(z, \tilde{z})$-superdifferentiable function $\chi$ such that $\left.\chi\right|_{W_{\xi}}=1, \operatorname{supp}(\chi)$ is a proper subset of $V_{\xi}$, consequently,

$\lim _{m \rightarrow \infty}\|\exp (m u(z))(\partial \chi(z) / \partial \tilde{z})\|_{C^{0}\left(U_{\alpha}\right)}=0$, where $(\partial \chi(z) / \partial \tilde{z})=\left(\partial \chi(z) / \partial^{1} \tilde{z}, \ldots, \partial \chi(z) / \partial^{n} \tilde{z}\right)$. In view of Theorem 3.10 there exist continuous functions $v_{m}$ on $\operatorname{cl}\left(U_{\alpha}\right)$ such that $\left(\partial v_{m} / \partial \tilde{z}\right)=\exp (m u(z))(\partial \chi / \partial \tilde{z})$ in $U_{\alpha}$ and $\lim _{m \rightarrow \infty}\left\|v_{m}\right\|_{C^{0}\left(U_{\alpha}\right)}=0$.

Put $g_{m}(z):=\exp (m u(z)) \chi(z)-v_{m}(z)+v_{m}(\xi)$, hence $g_{m}$ is continuous on $\operatorname{cl}\left(U_{\alpha}\right)$ and $\mathcal{A}_{p}$ holomorphic on $U_{\alpha}$. Since $\operatorname{supp}(\chi)$ is the proper subset in $V_{\xi}$, then $g_{m}(\xi)=1$ for each $m \in \mathbf{N}, \sup _{m}\left\|g_{m}\right\|_{C^{0}\left(U_{\alpha}\right)}<\infty$ and for each bounded closed subset $P$ in $\operatorname{cl}\left(U_{\alpha}\right) \backslash\{\xi\}$ there exists $\lim _{m}\left\|g_{m}\right\|_{C^{0}(P)}=0$. In view of Theorem 4.4.(ii) there exists a sequence of functions $f_{m} \in \mathcal{H}(M)$ and $C=$ const $<\infty$ such that $(a) f_{m}(\xi)=1$ for each $m \in \mathbf{N} ;(b)\left\|f_{m}\right\|_{C^{0}\left(U_{\alpha}\right)} \leq C$ for each $m \in \mathbf{N} ;(c) \lim _{m \rightarrow \infty}\left\|g_{m}\right\|_{C^{0}(P)}=0$ for each closed bounded subset $P \subset \operatorname{cl}\left(U_{\alpha}\right) \backslash\{\xi\}$.

Consider an $\mathcal{A}_{p}$ holomorphic function $f$ on a neighborhood of $\xi$ such that $f(\xi)=0$. Put $\phi_{m}:=f \exp (m u) \partial \chi / \partial \tilde{z}$, then $\operatorname{supp}\left(\phi_{m}\right)$ is the proper subset in $V_{\xi} \backslash W_{\xi}$. In view of Inequality (3) there exists $\delta>0$ such that $\lim _{m}\left\|\phi_{m}\right\|_{C^{0}\left(U_{\alpha+\delta}\right)}=0$. As in $\S 4.4$ it is possible to assume, that $\operatorname{Crit}(\rho)$ is discrete in $M$. Take $0<\epsilon<\delta$ such that $d \rho \neq 0$ on $\partial U_{\alpha+\epsilon}$. 
In view of Theorem 4.4.(ii) there exists a continuous function $v_{m}$ on $\operatorname{cl}\left(U_{\alpha+\epsilon}\right)$ such that $\partial v_{m} / \partial \tilde{z}=\hat{\phi}_{m}$ on $U_{\alpha+\epsilon}$ and $\lim _{m}\left\|v_{m}\right\|_{C^{0}\left(U_{\alpha+\epsilon}\right)}=0$. Each $v_{m}$ is $\mathcal{A}_{p}$-holomorphic on $W_{\xi}$, since $\phi_{m}=0$ on $W_{\xi}$, hence $\lim _{m} \partial v_{m}(\xi)=0$. Since $f(\xi)=u(\xi)=0$ and $\chi=1$ on $W_{\xi}$, then $\partial g_{k}(\xi) / \partial \xi=\partial f(\xi) / \partial \xi-\partial v_{k}(\xi) / \partial \xi$, where $g_{k}:=f(\chi \exp (k u))-v_{k}$. In view of Theorem 4.4. $(i)$ there exists $f_{m} \in \mathcal{H}(M)$ such that $\left\|f_{m}-g_{m}\right\|_{C^{0}\left(U_{\alpha+\epsilon}\right)}<m^{-1}$ and inevitably $\lim _{m}\left\|\partial f_{m}(\xi) / \partial \xi-\partial g_{m}(\xi) / \partial \xi\right\|=0$.

Let $V_{\xi}$ and $W_{\xi}$ be as above, then there exists $\delta>0$ such that $(u(z)+\tilde{u}(z)) / 2<-\delta$ for each $z \in U_{\alpha+\delta} \cap\left(V_{\xi} \backslash W_{\xi}\right)$. Therefore, there exists a branch of the $\mathcal{A}_{p}$ logarithm $\operatorname{Ln}(u) \in$ $\mathcal{H}\left(U_{\alpha+\delta} \cap\left(V_{\xi} \backslash \operatorname{cl}\left(W_{\xi}\right)\right)\right.$ (see $\left.\S \S 3.7,3.8[19,20]\right)$. From Theorems 4.2, 4.4 it follows that each $\mathcal{A}_{p}$ holomorphic Cousin problem over $U_{\alpha+\delta}$ has a solution. Hence $\operatorname{Ln}(u)=w_{1}-w_{2}$ for suitable $w_{1} \in \mathcal{H}\left(V_{\xi} \cap U_{\alpha+\delta}\right)$ and $w_{2} \in \mathcal{H}\left(U_{\alpha+\delta} \backslash c l\left(W_{\xi}\right)\right)$. Put $f:=u \exp \left(-w_{1}\right)$ in $U_{\alpha+\delta} \cap V_{\xi}$ and $f:=\exp \left(-w_{2}\right)$ in $U_{\alpha+\delta} \backslash \operatorname{cl}\left(W_{\xi}\right)$. Then $f \in \mathcal{H}\left(U_{\alpha+\delta}\right)$ and $f(\xi)=0$. In view of Inequality (3) $f(z) \neq 0$ for each $\xi \neq z \in \operatorname{cl}\left(U_{\alpha}\right)$. Verify now that $\operatorname{cl}\left(U_{\alpha}\right)$ is $\mathcal{H}(M)$-convex. Consider $\xi \in M \backslash \operatorname{cl}\left(U_{\alpha}\right)$. Due to $\S 4.4$ there exists a strictly plurisubharmonic exhausting $C_{z, \tilde{z}}^{\omega}$-function $\psi$ for $M$ such that $\operatorname{Crit}(\psi)$ is discrete and $U_{\alpha} \subset G_{\psi(\xi)}$, where $G_{\beta}:=\{z \in M: \psi(z)<\beta\}$ for $\beta \in \mathbf{R}$. Considering shifts $\psi \mapsto \psi+$ const assume $d \psi(z) \neq 0$ for each $z \in \partial G_{\psi(\xi)}$. From the proof above it follows, that there exists $f \in \mathcal{H}(M)$ such that $f(\xi)=1$ and $|f(z)|<1$ for each $z \in \operatorname{cl}\left(U_{\alpha}\right)$.

4.8.1. Remark. With the help of Theorem 4.8 it is possible to spread certain modifications of Theorems 3.2 and 3.5 on $\mathcal{A}_{p}$ Stein manifolds.

4.9. Theorem. Let $N$ be an $\mathcal{A}_{s}$ manifold with $1 \leq s \leq \infty$ (where $\mathcal{A}_{1}:=\mathrm{C}$ ), then for each $p$ with $s<p$ or $s \subset \mathbf{N} \subset p=\Lambda, s \neq p$, there exists an $\mathcal{A}_{p}$ manifold $M$ and an $\mathcal{A}_{s}$ holomorphic embedding $\theta: N \hookrightarrow M$.

Proof. Suppose $A t(N)=\left\{\left(V_{a}, \psi_{a}\right): a \in \Upsilon\right\}$ is any $\mathcal{A}_{s}$ holomorphic atlas of $N$, where $V_{a}$ is open in $N, \bigcup_{a} V_{a}=N, \psi_{a}: V_{a} \rightarrow \psi_{a}\left(V_{a}\right) \subset \mathcal{A}_{s}^{n}$ is a homeomorphism for each $a$, $n=\operatorname{dim}_{\mathcal{A}_{s}} M \in \mathbf{N},\left\{V_{a}: a \in \Upsilon\right\}$ is a locally finite covering of $N, \psi_{b} \circ \psi_{a}^{-1}$ is a holomorphic function on $\psi_{a}\left(V_{a} \cap V_{b}\right)$ for each $a, b \in \Lambda$ such that $V_{a} \cap V_{b} \neq \emptyset$. Since $\mathcal{A}_{p}^{n}$ is normed, then it is paracompact together with $M$ by Theorem 5.1.3 [6]. For each $\mathcal{A}_{s}$ holomorphic function $f$ on an open subset $V$ in $\mathcal{A}_{s}^{n}$ there exists an $\mathcal{A}_{p}$ holomorphic function $F$ on an open subset $U$ in $\mathcal{A}_{p}^{n}$ such that $\pi(U)=V$ and $\left.F\right|_{V}=\left.f\right|_{V}$, where $\pi: \mathcal{A}_{p}^{n} \rightarrow \mathcal{A}_{s}^{n}$ is the natural projection (see Proposition 3.13 [19] and analogously in the general case using local analyticity and a locally finite covering of $V$ ).

Therefore, for each two charts $\left(V_{a}, \psi_{a}\right)$ and $\left(V_{b}, \psi_{b}\right)$ with $V_{a, b}:=V_{a} \cap V_{b} \neq \emptyset$ there exists $U_{a, b}$ open in $\mathcal{A}_{p}^{n}$ and an $\mathcal{A}_{p}$ holomorphic function $\Psi_{b, a}$ such that $\left.\Psi_{b, a}\right|_{\psi_{a}\left(V_{a, b}\right)}=\left.\psi_{b, a}\right|_{\psi_{a}\left(V_{a, b}\right)}$, where $\psi_{b, a}:=\psi_{b} \circ \psi_{a}^{-1}, \pi\left(U_{a, b}\right)=\psi_{a}\left(V_{a, b}\right)$. Consider $Q:=\bigoplus_{a} Q_{a}$, where $Q_{a}$ is open in $\mathcal{A}_{p}^{n}, \pi\left(Q_{a}\right)=\psi_{a}\left(V_{a}\right)$ for each $a \in \Upsilon$. The equivalence relation $\mathcal{C}$ in the topological space $\bigoplus_{a} \psi_{a}\left(V_{a}\right)$ generated by functions $\psi_{b, a}$ has an extension to the equivalence relation $\mathcal{H}$ in $Q$. Then $M:=Q / \mathcal{H}$ is the desired $\mathcal{A}_{p}$ manifold with $\operatorname{At}(M)=\left\{\left(\Psi_{a}, U_{a}\right): a \in \Upsilon\right\}$ such that $\Psi_{b} \circ \Psi_{a}^{-1}=\Psi_{b, a}$ for each $U_{a} \cap U_{b} \neq \emptyset,\left.\Psi_{a}^{-1}\right|_{\psi_{a}\left(V_{a}\right)}=\left.\psi_{a}^{-1}\right|_{\psi_{a}\left(V_{a}\right)}$ for each $a, \Psi_{a}^{-1}: Q_{a} \rightarrow U_{a}$ is the $\mathcal{A}_{p}$ homeomorphism. Moreover, each homeomorphism $\psi_{a}: V_{a} \rightarrow \psi_{a}\left(V_{a}\right) \subset \mathcal{A}_{s}^{n}$ has the $\mathcal{A}_{p}$ extension up to the homeomorphism $\Psi_{a}: U_{a} \rightarrow \Psi_{a}\left(U_{a}\right) \subset \mathcal{A}_{p}^{n}$. The family of embeddings $\eta_{a}: \psi_{a}\left(V_{a}\right) \hookrightarrow Q_{a}$ such that $\pi \circ \eta_{a}=i d$ together with $A t(M)$ induces the $\mathcal{A}_{s}$ holomorphic embedding $\theta: N \hookrightarrow M$.

4.10. Definition. Let $M$ be an $\mathcal{A}_{p}$ manifold, $2 \leq p \in \mathbf{N}$ or $p=\Lambda$. Suppose that for each chart $\left(U_{a}, \phi_{a}\right)$ of $A t(M)$ there exists an $\mathcal{A}_{p}$ superdifferentiable mapping $\Gamma: u \in \phi_{a}\left(U_{a}\right) \mapsto$ $\Gamma(u) \in L_{q}\left(X, X, X_{q}^{*} ; \mathcal{A}_{p}\right)=L_{q}(X, X ; X)$, where $L_{q}\left(X^{n},\left(X_{q}^{*}\right)^{m} ; Y\right)$ denotes the space of all quasi-linear mappings from $X^{n} \times\left(X_{q}^{*}\right)^{m}$ into $Y$ (that is, additive and $\mathbf{R}$-homogeneous by each argument $x$ in $X$ or in $X_{q}^{*}$ ), where $X$ and $Y$ are Banach spaces over $\mathcal{A}_{p}, X_{q}^{*}$ denotes the space of all additive $\mathbf{R}$-homogeneous functionals on $X$ with values in $\mathcal{A}_{p}$ (see $\S 4.1$ ), 
$X_{q}^{*}=L_{q}\left(X ; \mathcal{A}_{p}\right)$. If $U_{a} \cap U_{b} \neq \emptyset$, let

(1) $D\left(\phi_{b} \circ \phi_{a}^{-1}\right) \cdot \Gamma\left(\phi_{a}\right)=D^{2}\left(\phi_{b} \circ \phi_{a}^{-1}\right)+\Gamma\left(\phi_{b}\right) \circ\left(D\left(\phi_{b} \circ \phi_{a}^{-1}\right) \times D\left(\phi_{b} \circ \phi_{a}^{-1}\right)\right)$. These $\Gamma\left(\phi_{a}\right)$ are called the Christoffel symbols. Let $\mathcal{B}=\mathcal{B}(M)$ be a family of all $\mathcal{A}_{p}$ holomorphic vector fields on $M$. For $M$ supplied with $\left\{\Gamma\left(\phi_{a}\right): a\right\}$ define a covariant derivation $(X, Y) \in \mathcal{B}^{2} \mapsto$ $\nabla_{X} Y \in \mathcal{B}:$

$(2) \nabla_{X} Y(u)=D Y(u) \cdot X(u)+\Gamma(u)(X(u), Y(u))$, where $X(u)$ and $Y(u)$ are the principal parts of $X$ and $Y$ on $\left(U_{a}, \phi_{a}\right), u=\phi_{a}(z), z \in U_{a}$. In this case it is said that $M$ possesses a covariant derivation.

4.11. Remark. Certainly for an $\mathcal{A}_{p}$ manifold there exists a neighbourhood $V$ of $M$ in $T M$ such that $\exp : V \rightarrow M$ is $\mathcal{A}_{p}$ holomorphic (see the real case in [9]).

4.12. Theorem. Let $f$ be an $\mathcal{A}_{p}$ holomorphic function such that $\hat{f}$ is $\mathcal{A}_{p}$ (right) superlinear on a compact $\mathcal{A}_{p}$ manifold $M$, where $2 \leq p \in \mathbf{N}$. Then $f$ is constant on $M$.

Proof. By the supposition of this theorem $\left(f \circ \phi_{b}^{-1}\right) \hat{\text {. is }} \mathcal{A}_{p}$ (right) superlinear for each chart $\left(U_{b}, \phi_{b}\right)$ of $M$. Since $M$ is compact and $|f(z)|$ is continuous, then there exists a point $q \in M$ at which $|f(z)|$ attains its maximum. Let $q \in U_{b}$, then $f \circ \phi_{b}^{-1}$ is the $\mathcal{A}_{p}$ holomorphic function on $V_{b}:=\phi_{b}\left(U_{b}\right) \subset \mathcal{A}_{p}^{n}$, where $\operatorname{dim}_{\mathcal{A}_{p}} M=n$. Consider a polydisk $V$ in $\mathcal{A}_{p}^{n}$ with the centre $y=\phi_{b}(q)$ such that $V \subset V_{b}$. Put $g(w)=f \circ \phi_{b}^{-1}(y+(z-y) w)$, where $w$ is the $\mathcal{A}_{p}$ variable. Then for each $z \in V$ there exists $\epsilon_{z}>0$ such that the function $g(w)$ is $\mathcal{A}_{p}$ holomorphic on the set $W_{z}:=\left\{w: w \in \mathcal{A}_{p},|w|<1+\epsilon_{z}\right\}$ and $|g(w)|$ attains its maximum at $w=0$. In view of Theorem 3.15 and Remark $3.16[19,20] g$ is constant on $W_{z}$, hence $f$ is constant on $U_{b}$. By the $\mathcal{A}_{p}$ holomorphic continuation $f$ is constant on $M$.

\section{References}

[1] J.C. Baez. "The octonions". Bull. Amer. Mathem. Soc. 39: 2 (2002), 145-205.

[2] F.A. Berezin. "Introduction to superanalysis" (D. Reidel Publish. Comp., Kluwer group: Dordrecht, 1987).

[3] A. Connes. "Noncommutative geometry" (Academic Press: San Diego, 1994).

[4] B. DeWitt. "Supermanifolds" 2d ed. (Cambridge Univ. Press: Cambridge, 1992).

[5] G. Emch. "Mécanique quantique quaternionienne et Relativité restreinte", Helv. Phys. Acta 36 (1963), 739-769.

[6] R. Engelking. "General topology" (Heldermann: Berlin, 1989).

[7] G.M. Henkin, J. Leiterer. "Theory of functions on complex manifolds" (Series "Monographs in Mathematics"; V. 79; Birkhäuser: Basel, 1984).

[8] A. Khrennikov. "Superanalysis", (Series "Mathem. and its Applic."; V. 470; Kluwer: Dordrecht, 1999).

[9] W. Klingenberg. "Riemannian geometry" (Walter de Gruyter: Berlin, 1982).

[10] K. Kodaira. "Complex manifolds and deformation of complex structures" (SpringerVerlag: New York, 1986).

[11] A.N. Kolmogorov, S.V. Fomin. "Reele Funktionen und Funktionalanalysis" (VEB Deutscher Verlag der Wissenschaften: Berlin, 1975). 
[12] A.G. Kurosh. "Lectures in general algebra" (Nauka: Moscow, 1973).

[13] H.B. Lawson, M.-L. Michelsohn. "Spin geometry" (Princeton Univ. Press: Princeton, New Jersey, 1989).

[14] J.J. Loeb, M. Nicolau, "Holomorhpic Flows and Complex Structures on Products of Odd-Dimensional Spheres", Mathem. Annalen, 306 (1996), 781-817.

[15] S.V. Lüdkovsky. "Generalized Geometric Loop Groups of Complex Manifolds, Gaussian Quasi-Invariant Measures on them and their Representations". J. of Math. Sciences, 122: 1 (2004), 2984-3010.

[16] S.V. Lüdkovsky. "Gaussian measures on free loop spaces". Russian Mathem. Surveys (Usp. Mat. Nauk). 56 : 5 (2001), 183-184.

[17] S.V. Lüdkovsky. "Stochastic processes on groups of diffeomorphisms and loops of real, complex and non-Archimedean manifolds". Fundam. i Prikl. Mathem. 7: 4 (2001), 10911105 (see also Los Alamos National Laboratory, USA. Preprint math.GR/0102222, 35 pages, 28 February 2001).

[18] S.V. Lüdkovsky. "Poisson measures for topological groups and their representations". Southeast Asian Bull. Math. 25: 4 (2002), 653-680.

[19] S.V. Lüdkovsky, F.van Oystaeyen. "Differentiable functions of quaternion variables". Bull. Sci. Math. (Paris). Ser. 2. 127 (2003), 755-796.

[20] S.V. Lüdkovsky. "Differentiable functions of Cayley-Dickson numbers", Los Alamos Nat. Lab., USA. Preprint math.CV/0405471, 63 pages, 26 July 2004.

[21] J.D. Moore. "Lectures on Seiberg-Witten invariants" (Springer-Verlag: Berlin, 1996).

[22] N. Murakoshi, K. Sekigawa, A. Yamada. "Integrability of almost quaternionic manifolds". Indian J. Mathem. 42: 3 (2000), 313-329.

[23] L. Narici, E. Beckenstein. "Topological vector spaces" (Marcel-Dekker Inc.: New York, 1985).

[24] F. van Oystaeyen. "Algebraic geometry for associative algebras" (Series "Lect. Notes in Pure and Appl. Mathem."; V. 232; Marcel Dekker: New York, 2000).

[25] Yu.P. Razmyslov. "Identities of algebras and their representations" (Series "Modern Algebra"; V. 14; Nauka: Moscow, 1989).

[26] E.H. Spanier. "Algebraic topology" (Acad. Press: New York, 1966).

[27] V.A. Zorich." Mathematical Analysis" (Nauka: Moscow, 1984).

Address: Sergey V. Ludkovsky, Mathematical Department, Brussels University, Pleinlaan 1, Brussels, Belgium.

Acknowledgment. The author thanks the Flemish Science Foundation for support through the Noncommutative Geometry from Algebra to Physics project and Professors Stefaan Caenepeel and Fred van Oystaeyen for hospitality. 